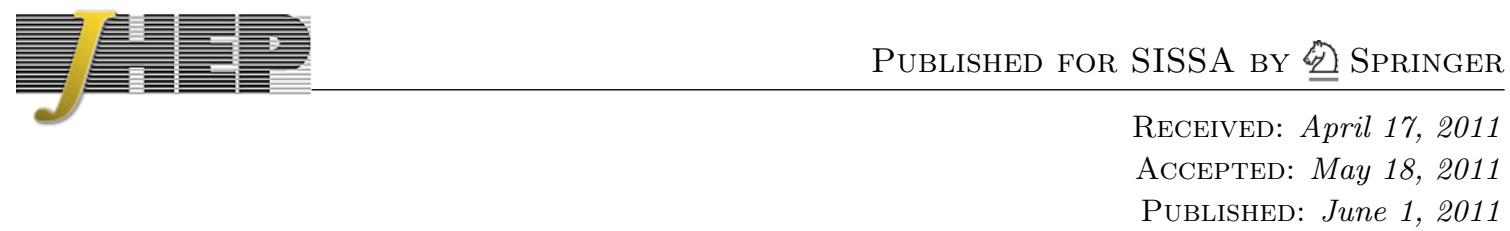

\title{
Spin-2 spectrum of defect theories
}

\author{
Constantin Bachas ${ }^{a}$ and John Estes ${ }^{b}$ \\ ${ }^{a}$ Laboratoire de Physique Théorique de l'Ecole Normale Supérieure, \\ 24 rue Lhomond, 75231 Paris cedex, France \\ ${ }^{b}$ Instituut voor Theoretische Fysica, Katholieke Universiteit Leuven, \\ Celestijnenlaan 200D B-3001 Leuven, Belgium \\ E-mail: bachas@lpt.ens.fr, johnalondestes@gmail.com
}

ABSTRACT: We study spin-2 excitations in the background of the recently-discovered typeIIB solutions of D'Hoker et al. These are holographically-dual to defect conformal field theories, and they are also of interest in the context of the Karch-Randall proposal for a string-theory embedding of localized gravity. We first generalize an argument by Csaki et al to show that for any solution with four-dimensional anti-de Sitter, Poincaré or de Sitter invariance the spin-2 excitations obey the massless scalar wave equation in ten dimensions. For the interface solutions at hand this reduces to a Laplace-Beltrami equation on a Riemann surface with disk topology, and in the simplest case of the supersymmetric Janus solution it further reduces to an ordinary differential equation known as Heun's equation. We solve this equation numerically, and exhibit the spectrum as a function of the dilaton-jump parameter $\Delta \phi$. In the limit of large $\Delta \phi$ a nearly-flat linear-dilaton dimension grows large, and the Janus geometry becomes effectively five-dimensional. We also discuss the difficulties of localizing four-dimensional gravity in the more general backgrounds with NS5-brane or D5-brane charge, which will be analyzed in detail in a companion paper.

KeYwords: Gauge-gravity correspondence, Large Extra Dimensions, AdS-CFT Correspondence, Field Theories in Higher Dimensions

ARXIV EPRINT: 1103.2800 


\section{Contents}

1 Introduction 1

2 Universal equation for spin-2 excitations 3

2.1 Ansatz for the Kaluza-Klein spin-2 modes 3

2.2 Reduction of the Einstein equations 4

2.3 Normalizability and the zero mode 8

3 Interface solutions and their charges $\quad 9$

3.1 Local solutions: general form $r$

$\begin{array}{lll}3.2 & \mathrm{AdS}_{5} \times \mathrm{S}^{5} \text { and supersymmetric Janus } & 11\end{array}$

$\begin{array}{lll}3.3 & \text { Non-vanishing five-brane charge } & 13\end{array}$

4 Localized gravity: what to look for? $\quad 16$

$\begin{array}{ll}4.1 \text { The Karch-Randall model } & 16\end{array}$

$\begin{array}{ll}4.2 \text { Warp factors and a limiting geometry } & 19\end{array}$

5 Spectral problem on the strip $\quad 21$

5.1 Reduction of the eigenmode equation 22

5.2 The case of $\mathrm{AdS}_{5} \times \mathrm{S}^{5} \quad 25$

6 Spectrum of supersymmetric Janus 26

6.1 Heun's equation 26

$\begin{array}{ll}6.2 \text { Numerical solution } & 28\end{array}$

$\begin{array}{lll}7 & \text { Discussion } & 30\end{array}$

$\begin{array}{ll}\text { A Summary of type IIB supergravity } & 31\end{array}$

\section{Introduction}

Whether gravity can be localized [1-3] is a question of great interest for cosmology. It is closely related to the question of whether the graviton can have a mass [4-8], or more generally whether Einstein's theory - with or without a cosmological constant term can be consistently modified at cosmic-distance scales. Despite many interesting results (see e.g. [9-16] and references therein), there is still no definitive answer to these questions.

The difficulties with low-energy descriptions of localized and/or massive gravity typically arise when one considers classical non-linearities, and quantum corrections. Problems include the non-decoupling of heavy mass scales (the Planck scale, the bulk curvature or the brane thickness) and/or the appearance of ghosts. Clearly, an embedding in an 
ultraviolet-complete theory like string theory could shed important light on these issues. A promising proposal for such an embedding was made some time ago by Karch and Randall [17, 18]. These authors considered an $\mathrm{AdS}_{4} \times \mathrm{S}^{2}$ brane in an $\mathrm{AdS}_{5} \times \mathrm{S}^{5}$ bulk, and argued that a nearly-massless graviton can be obtained by tuning the brane tension. In their calculation Karch and Randall had to approximate the background geometry by two $\mathrm{AdS}_{5}$ slices glued together along a "thin" $\mathrm{AdS}_{4}$ brane. The exact type-IIB supergravity solutions, describing the full back-reaction of branes on geometry, were discovered only recently by D'Hoker et al [19, 20] (see also [21, 22] for related work). A central motivation for our work here was to check whether the conclusions of Karch and Randall survive in these exact string-theory backgrounds.

The supergravity solutions of D'Hoker et al are holographically-dual to defect conformal field theories [23-29], i.e. to two or more conformal theories interacting along a scale-invariant domain wall. ${ }^{1}$ The "locally-localized graviton" has been discussed from the viewpoint of the defect CFTs in [32] (for earlier perspectives see [33-36], and for a recent one [37] ). Other than their potential relevance to the localization of gravity, our results are thus also of interest in this dual context.

In this paper we will first set up the spectral problem for spin-2 excitations in the interface geometries of refs. [19, 20]. These have the structure of an $\mathrm{AdS}_{4} \times \mathrm{S}^{2} \times \mathrm{S}^{2}$ spacetime, fibered over a Riemann surface $(\Sigma, \hat{g})$ with disk topology. The excitations which are constant on the spheres, and which have spin 2 in $\mathrm{AdS}_{4}$, obey Neumann boundary conditions on the surface $\Sigma$. Their shifted mass-squared operator, $m^{2}+2$, is, as we will see, the Laplace-Beltrami operator on the disk with metric given by $\bar{g}=e^{-2 A} \hat{g}$, where $A$ is the $\mathrm{AdS}_{4}$ warping factor. In deriving this result, we will generalize slightly an argument of Csaki et al [38] to show that the spin-2 excitations obey the massless scalar-wave equation in ten dimensions. The argument, first made in the context of flat thick branes and scalar fields, ${ }^{2}$ generalizes easily to all supergravity solutions with 4D anti-de Sitter, Poincaré or de Sitter symmetry, and with arbitrary $p$-form flux backgrounds. The universality of the spin-2 wave equation should be contrasted with what happens for spins $\leq 1$, whose wave equations depend a priori on non-metric features of the backgrounds.

Solving the spectral problem on the surface $(\Sigma, \bar{g})$ is, in general, a difficult task. It simplifies considerably for the supersymmetric Janus solution [which describes a supersymmetric dilaton domain wall], for which the relevant eigenmode equation is separable. The reduced ordinary differential equation has four regular singular points, and is known in the mathematics literature as Heun's equation [40]. We will solve this equation numerically, and exhibit the spectrum as a function of the dilaton-jump parameter $\Delta \phi$. As we will see, Janus cannot localize four-dimensional gravity and its only interesting limit, $\Delta \phi \rightarrow \infty$, is a limit in which a flat fifth dimension decompactifies. This behavior can be attributed to the vanishing superpotential of the dilaton field, whose domain walls have no intrinsic tension or thickness. Solutions with NS5-brane and/or D5-brane charge are in this respect

\footnotetext{
${ }^{1}$ To be distinguished from conformal theories interacting via multi-trace marginal operators in the bulk, and which have been argued to be the duals of multi-gravity models [13, 30, 31]. Their embedding in string theory is more problematic, as it most likely involves quantum-gravity effects.

${ }^{2}$ For branes of co-dimension one, the observation was actually made earlier in ref. [39].
} 
more promising, in line with the original proposal of Karch and Randall [18]. Generating a large scale hierarchy without decompactifying extra dimensions looks, however, a priori a difficult task. The detailed analysis of the backgrounds with five-brane charge, for which the eigenmode equation on the strip does not factorize, will be presented in a forthcoming companion publication [41].

This paper is organized as follows: in section 2 we derive the linearized equation for transverse-traceless graviton modes in any geometry with AdS, dS or Poincaré invariance in four dimensions. Generalizing the argument of [38] we show that, in all cases, these modes obey the massless scalar wave equation in ten dimensions. In section 3 we review the solutions of D'Hoker et al [19, 20], and relate their free parameters to brane charges and to the asymptotic values of the dilaton field. Section 4 reviews the qualitative features responsible for the localization of the graviton in the protoype thin-brane geometry of Karch and Randall [17]. We show that the Janus solution does not have the required "warp-factor bump", and we describe the large- $\Delta \phi$ limit of its geometry. We also discuss briefly the backgrounds with five-brane charge, whose detailed analysis will be presented in ref. [41]. In section 5 we reduce the eigenmode equation for all interface solutions to a spectral problem on the two-dimensional strip $\Sigma$, paying special attention to the boundary conditions and the norm of the modes. We verify our formulae by rederiving the wellknown $\mathrm{AdS}_{5} \times \mathrm{S}^{5}$ spectrum. Finally, section 6 presents our analysis of the Janus spectrum. We reduce the equation on $\Sigma$ to Heun's equation [40], and solve this latter numerically. The results are not surprising, and agree with expectations in the large- $\Delta \phi$ limit. Some conventions on type-IIB supergravity are collected in the appendix A.

\section{Universal equation for spin-2 excitations}

In this section we will derive the equation for the spectrum of the spin-2 Kaluza-Klein excitations around any solution with maximal symmetry in four dimensions. As we will see, the relevant second-order operator is the massless scalar wave operator in ten (or in eleven) dimensions. It is an interesting fact that this mass operator only depends on the background metric, and on no other fields. The reason is that all other background fields enter the linearized equations for spin-2 modes via the total energy-momentum tensor, which can be re-expressed in terms of the background curvature. This was first observed by Csaki et al [38] for flat thick branes generated by scalar fields. We here extend the argument to all supergravity solutions with maximal (AdS, dS or Poincaré) symmetry, and with arbitrary $p$-form flux backgrounds.

\subsection{Ansatz for the Kaluza-Klein spin-2 modes}

We are interested in solutions of the supergravity equations with a background metric that has the following warped form:

$$
\widehat{d s^{2}}=e^{2 A(y)} \bar{g}_{\mu \nu}(x) d x^{\mu} d x^{\nu}+\hat{g}_{a b}(y) d y^{a} d y^{b} .
$$

Here Greek indices run over $\{0,1,2,3\}, \bar{g}_{\mu \nu}$ is the metric for the unit-radius constantcurvature spacetime $\left(\overline{\mathcal{M}}_{4}=\mathrm{AdS}_{4}, \mathbb{M}_{4}\right.$ or $\mathrm{dS}_{4}$, for $\left.k=-1,0,1\right)$, and Latin indices label 
the remaining coordinates, $y^{a}$, of the "internal" space $\hat{\mathcal{M}}_{d-4}$. The internal space may, but need not, be compact. In this section the total spacetime dimension is arbitrary, later we will focus on the type IIB supergravity for which $d=10$.

We restrict attention to perturbations of the four-dimensional metric:

$$
d s^{2}=e^{2 A}\left(\bar{g}_{\mu \nu}+h_{\mu \nu}\right) d x^{\mu} d x^{\nu}+\hat{g}_{a b} d y^{a} d y^{b},
$$

and look for factorized solutions of the linearized field equations of the following form:

$$
h_{\mu \nu}(x, y)=h_{\mu \nu}^{[\mathrm{tt}]}(x \mid \lambda) \psi(y \mid \lambda)
$$

where $h_{\mu \nu}^{[\mathrm{tt}]}$ solves the Pauli-Fierz equations $[42,43]$ for a massive spin-2 particle in $\overline{\mathcal{M}}_{4}$. The Pauli-Fierz equations read (see for instance [44, 45])

$$
\left(\bar{\square}_{x}^{(2)}-\lambda\right) h_{\mu \nu}^{[\mathrm{tt}]}=0 \quad \text { and } \quad \bar{\nabla}^{\mu} h_{\mu \nu}^{[\mathrm{tt}]}=\bar{g}^{\mu \nu} h_{\mu \nu}^{[\mathrm{tt}]}=0,
$$

where $\bar{\square}_{x}^{(2)}$ is the Laplace operator acting on two-index tensors in $\overline{\mathcal{M}}_{4}$. The last two equations, which force $h_{\mu \nu}^{[\mathrm{tt}]}$ to be transverse and traceless (henceforth " $\mathrm{t}$ ") in four dimensions, are non-dynamical phase-space constraints. As we will show, with the above ansatz the linearized Einstein equations reduce to a single second-order equation for the internal-space wavefunction $\psi(y)$. Normalizable solutions correspond to the allowed eigenvalues of the wave operator, $\lambda$. The four-dimensional Pauli-Fierz mass is related to $\lambda$ as $m^{2}=\lambda-2 k$ [45].

The last two equations in (2.4) eliminate five out of the ten components of the symmetric two-index tensor. The remaining degrees of freedom are what one needs to describe the states of a massive spin- 2 particle in four dimensions. We note that the above metric perturbations are transverse-traceless also in $d$ dimensions,

$$
\hat{\nabla}^{M} \delta g_{M N}=\hat{g}^{M N} \delta g_{M N}=0,
$$

where here the covariant derivatives refer to the (bloc-diagonal) metric $\hat{g}_{M N}=\left(e^{2 A} \bar{g}_{\mu \nu}, \hat{g}_{a b}\right)$, and $\delta g_{M N}=\left(e^{2 A} h_{\mu \nu}, 0\right)$ is the unscaled metric perturbation. To verify this statement one uses the fact that the components $\hat{\Gamma}_{\mu \nu}^{\rho}$ of the affine connection are identical to those on $\overline{\mathcal{M}}_{4}$. The converse of this statement is however false, i.e. the $d$-dimensional transverse-traceless conditions do not imply the four-dimensional ones. Thus our ansatz does not describe all metric perturbations, but only those which have spin 2 in four dimensions.

\subsection{Reduction of the Einstein equations}

Turning now to the Einstein equations, we want to compute the linearized variation of the Einstein tensor and of its source term, i.e. the energy-momentum tensor of the matter fields. Let us start with the Einstein tensor. The variations of the affine connection and of the curvature are given, at linear order, by the expressions [46]:

$$
\begin{aligned}
\delta \Gamma_{M N}^{K} & =\frac{1}{2} \hat{g}^{K \Sigma}\left(\hat{\nabla}_{M} \delta g_{N \Sigma}+\hat{\nabla}_{N} \delta g_{M \Sigma}-\hat{\nabla}_{\Sigma} \delta g_{M N}\right), \\
\delta R_{M K N}^{P} & =\hat{\nabla}_{K}\left(\delta \Gamma_{N M}^{P}\right)-\hat{\nabla}_{N}\left(\delta \Gamma_{K M}^{P}\right) .
\end{aligned}
$$


Using the [tt] property of $\delta g_{M N}$ leads, after a little algebra, to the following linear variation of the Ricci tensor:

$$
\begin{aligned}
\delta R_{M N} & =\frac{1}{2}\left[\hat{\nabla}_{K}, \hat{\nabla}_{M}\right] \delta g_{N}^{K}+\frac{1}{2}\left[\hat{\nabla}_{K}, \hat{\nabla}_{N}\right] \delta g_{M}^{K}-\frac{1}{2} \hat{\nabla}_{K} \hat{\nabla}^{K} \delta g_{M N} \\
& =\frac{1}{2}\left(\hat{R}_{P M} \delta g_{N}^{P}-\hat{R}_{N K M}^{P} \delta g_{P}^{K}+(N \leftrightarrow M)\right)-\frac{1}{2} \hat{\nabla}^{K} \hat{\nabla}_{K} \delta g_{M N}
\end{aligned}
$$

where indices are raised with the background metric $\hat{g}_{M N}$. It is at this stage convenient to bring the metric by a Weyl rescaling to direct-product form [we use bars for the rescaled background, as opposed to hats for the original one]:

$$
\bar{g}_{M N} \equiv e^{-2 A} \hat{g}_{M N}=\left(\bar{g}_{\mu \nu}, e^{-2 A} \hat{g}_{a b}\right)
$$

The curvature and Ricci tensors in (2.7) can be expressed in terms of the corresponding "barred" tensors, and derivatives of the conformal factor (see e.g. [47] for a collection of Weyl-transformation formulae). The terms relevant for our purposes here are:

$$
\begin{aligned}
\hat{R}_{N K M}^{P} & =\bar{R}_{N K M}^{P}+\left(\bar{g}_{M}^{P} \bar{g}_{N K}-\bar{g}_{K}^{P} \bar{g}_{N M}\right) A_{; \Sigma} A^{; \Sigma}+\cdots, \\
\hat{R}_{P M} & =\bar{R}_{P M}-\bar{g}_{P M}\left[\square_{y} A+(d-2) A_{; \Sigma} A^{; \Sigma}\right]+\cdots
\end{aligned}
$$

where $d$ is the total spacetime dimension, $\bar{\square}_{y}$ is the scalar Laplacian in the internal space with metric $\bar{g}_{a b}=e^{-2 A} \hat{g}_{a b}$, and $A_{; \Sigma} \equiv \partial_{\Sigma} A$. The dots stand for omitted terms that vanish when inserted in (2.7), either because the perturbation $\delta g_{M N}$ is traceless, or because it points in the $\overline{\mathcal{M}}_{4}$ directions (so that its contraction with $A_{; M}$ is zero). Note that the second line in (2.9) is not just the contraction of the first line, because the terms omitted in these two lines are different.

The Weyl transformation of the third term in expression (2.7) requires some more work. From the transformation property of the affine connection,

$$
\hat{\Gamma}_{M N}^{K}=\bar{\Gamma}_{M N}^{K}+\left(\bar{g}_{M}^{K} A_{; N}+\bar{g}_{N}^{K} A_{; M}-\bar{g}_{M N} A^{; K}\right),
$$

and the fact that in our case $A^{; \Sigma} \delta g_{\Sigma \Xi}=0$ one finds first:

$$
\begin{aligned}
\hat{\nabla}_{K} \delta g_{M N} & =\bar{\nabla}_{K} \delta g_{M N}-2 A_{; K} \delta g_{M N}-A_{; M} \delta g_{K N}-A_{; N} \delta g_{M K} \\
& =e^{2 A}\left(\bar{\nabla}_{K} h_{M N}-A_{; M} h_{K N}-A_{; N} h_{M K}\right)
\end{aligned}
$$

where $h_{M N} \equiv e^{-2 A} \delta g_{M N}$ is the rescaled perturbation. Applying one more derivative, and using again the special properties of $h_{M N}$, gives after some straightforward algebra,

$$
\hat{\nabla}^{K} \hat{\nabla}_{K} \delta g_{M N}=\bar{\nabla}^{K} \bar{\nabla}_{K} h_{M N}+(d-2) A_{; K} \bar{\nabla}^{K} h_{M N}-2 A_{; \Sigma} A^{; \Sigma} h_{M N}
$$

Inserting now the expressions (2.9) and (2.12) in the second line of (2.7) gives the variation of the Ricci tensor, expressed in terms of the rescaled metric $\bar{g}_{M N}$, its curvature tensor, and the warp factor $A(y)$. The advantage of this rewriting is that $\bar{g}_{M N}$ is a directproduct metric, so that its curvature tensor $\bar{R}_{P N K M}$ is bloc-diagonal, i.e. either all indices 
are in $\overline{\mathcal{M}}_{4}$ or they are all in the internal-space directions. For our purposes here, we only need the curvature tensor with indices in $\overline{\mathcal{M}}_{4}$. It reads

$$
\bar{R}_{\rho \nu \kappa \mu}=k\left(\bar{g}_{\rho \kappa} \bar{g}_{\nu \mu}-\bar{g}_{\rho \mu} \bar{g}_{\kappa \nu}\right),
$$

where $k=-1,0,1$ for anti-de Sitter, Minkowski or de Sitter spacetime. Furthermore, when acting on the factorized perturbation $h_{\mu \nu}(x, y) \equiv h_{\mu \nu}^{[\mathrm{tt}]}(x) \psi(y)$, the wave operator breaks down to a direct sum: $\bar{\nabla}^{K} \bar{\nabla}_{K}=\bar{\square}_{x}^{(2)}+\bar{\square}_{y}$. Putting everything together, and using also the four-dimensional wave equation (2.4), leads to the following linear variation of the Ricci tensor with indices in $\{0,1,2,3\}$ :

$$
\delta R_{\mu \nu}=-\frac{1}{2} h_{\mu \nu}^{[t t]}\left[\lambda-8 k+(2 d-4)(\partial A)^{2}+2\left(\bar{\square}_{y} A\right)+(d-2)\left(\partial_{a} A\right) \partial^{a}+\bar{\square}_{y}\right] \psi,
$$

where internal indices are contracted with the metric $\bar{g}_{a b}$. Furthermore, because $h_{\mu \nu}^{[t t]}$ is traceless the variation of the Ricci scalar vanishes, so that

$$
\delta\left[g_{\mu \nu} R\right]=\hat{R} \delta g_{\mu \nu}=h_{\mu \nu}^{[t t]}(x) \psi(y) e^{2 A} \hat{R} .
$$

This completes the computation of the linear variation of the Einstein tensor in the $\{0,1,2,3\}$ directions. As the reader can readily verify, the variation of the remaining components of the Einstein tensor vanishes identically.

We turn next to the variation of the energy-momentum tensor of "matter" fields, i.e. of all fields other than the $d$-dimensional graviton. These include the scalar and $p$-form gauge fields, which by abuse of notation we will refer to collectively as $\Phi$. In general, the perturbations $\delta \Phi$ mix at the linearized level with metric perturbations. Put differently, the Lagrangian expanded to quadratic order around the background solution, may contain terms $\sim \delta g_{M N} \delta \Phi$. This cannot however happen in the case at hand, because the special form of metric perturbations and the maximal symmetry of $\overline{\mathcal{M}}_{4}$ forbid all contractions of $\delta g_{M N}$ with any field other than itself. ${ }^{3}$ Indeed, background fields must be proportional to invariant $\mathrm{AdS}_{4}$ tensors and neither the metric nor covariant derivatives can contract with $\delta g_{\mu \nu}$. Thus, it is consistent to set $\delta \Phi=0$ when computing the linearized equations for spin-2 modes.

The energy-momentum tensor of the matter fields is given by the variation of their Lagrangian density,

$$
T_{M N}=\frac{2}{\sqrt{g}} \frac{\partial}{\partial g^{M N}}\left(\sqrt{g} \mathcal{L}_{\text {mat }}\right)
$$

By our previous argument, we need only consider $\mathcal{L}_{\text {mat }}$ for $\Phi=\hat{\Phi}$. But thanks to the symmetry of the background fields, $\mathcal{L}_{\text {mat }}\left(g_{M N}, \hat{\Phi}\right)=\mathcal{L}_{\text {mat }}\left(\operatorname{det}\left(g_{\mu \nu}\right), g_{a b}, \hat{\Phi}\right)$, i.e. the Lagrangian density may depend on the 4-dimensional part of the metric only via its determinant. Indeed, the only allowed background fields with indices in $\{0,1,2,3\}$ are $p$-form field strengths proportional to the $\epsilon$ symbol, and whose contraction in $\mathcal{L}_{\text {mat }}$ gives a factor of $\operatorname{det}\left(g_{\mu \nu}\right)$. The

\footnotetext{
${ }^{3}$ This argument does not of course exclude cubic or higher-order mixing terms.
} 
reader can also verify that $4 \mathrm{D}$ space-time filling sources, such as D-branes or orientifolds, do not modify the above conclusion. It follows that at linear order $T_{\mu \nu} \propto g_{\mu \nu}$, where the scalar proportionality factor is made out of background fields. Consistency of the trace relation then implies

$$
T_{\mu \nu}=\left(\hat{g}_{\mu \nu}+\delta g_{\mu \nu}\right) \frac{1}{4} \hat{T}_{\rho}{ }^{\rho}+\mathcal{O}\left(\delta g^{2}\right)
$$

where $\hat{T}_{\rho \sigma}$ is the energy-momentum tensor of the background solution. By similar arguments one shows that $\delta T_{a b}=\delta T_{a \nu}=0$ at the linear level, in agreement with the fact that the corresponding components of the Einstein tensor vanish.

To complete our calculation we now use the Einstein equations in order to express the partial trace $\hat{T}_{\rho}{ }^{\rho}$ as follows:

$$
\hat{T}_{\rho}^{\rho}=\hat{R}_{\rho}^{\rho}-2 \hat{R}=12 k e^{-2 A}-4 e^{-2 A}\left(\bar{\square}_{y} A+(d-2)(\partial A)^{2}\right)-2 \hat{R},
$$

where in the second equality we have used once more the Weyl transformation of the Ricci tensor. Combining finally $(2.14),(2.15),(2.17)$ and (2.18) leads to the following equation for the wavefunction of spin-2 excitations in the internal space:

$$
-\left[\bar{\square}_{y}+(d-2) \bar{g}^{a b}\left(\partial_{a} A\right) \partial_{b}\right] \psi=m^{2} \psi,
$$

where we recall that $m^{2}=\lambda-2 k$. The above eigenvalue equation is the main result of this section. It confirms and generalizes the analysis of Csaki et al [38]. As advertized earlier the linearized equation only involves the background metric, but no other details of the background fields.

There are several useful rewritings of this equation. Firstly, in terms of the original internal-space metric $\hat{g}_{a b}=e^{2 A} \bar{g}_{a b}$, it reads

$$
-\frac{e^{-2 A}}{\sqrt{[\hat{g}]}}\left(\partial_{a} \sqrt{[\hat{g}]} \hat{g}^{a b} e^{4 A} \partial_{b}\right) \psi=m^{2} \psi
$$

where $[\hat{g}]$ is the determinant of $\hat{g}_{a b}$ (we reserve the notation $\hat{g}$ for the determinant of the full $d$-dimensional metric $\left.\hat{g}_{M N}\right)$. Alternatively, one can put it in the form of a Schröndinger-like problem in the internal space:

$$
\left[-\square_{y}+V(y)\right] \Psi=m^{2} \Psi, \quad \text { where } \Psi=e^{\frac{1}{2}(d-2) A} \psi
$$

and the "analog potential" is given by

$$
V(y)=e^{-\frac{1}{2}(d-2) A} \square_{y} e^{\frac{1}{2}(d-2) A} .
$$

A third useful rewriting is as a wave equation in $d$ dimensions. One first verifies that the identity $m^{2}=\bar{\square}_{x}^{(2)}-2 k=\bar{\square}_{x}$ is valid for operators acting on transverse-traceless tensor fields in the maximally-symmetric spacetime $\overline{\mathcal{M}}_{4}$ (see e.g. ref. [48]). The spin-2 equation takes then a simple and universal form, as the scalar Laplace-Beltrami ("box") equation in the full $d$-dimensional spacetime:

$$
\frac{1}{\sqrt{\hat{g}}}\left(\partial_{M} \sqrt{\hat{g}} \hat{g}^{M N} \partial_{N}\right) h_{\mu \nu}(x, y)=0 .
$$


Note that the universality of the spin-2 mass operator should be contrasted with what happens for lower-spin fields, whose linearized equations typically depend on non-metric details of the background solution.

\subsection{Normalizability and the zero mode}

The eigenmode equation must be supplemented with a space of admissible $\psi(y)$, which requires the introduction of a norm. To avoid cumbersome notation, let us first consider the case of a scalar field and look for factorized solutions of its wave equation of the form $\delta \Phi(x, y)=\phi(x) \psi(y)$. Assuming a canonical kinetic term, these solutions are normalized by fixing the following invariant integral:

$$
\|\delta \Phi\|^{2} \equiv i \int_{\mathcal{S}} d S^{M}\left(\delta \Phi_{-} \partial_{M} \delta \Phi_{+}-\delta \Phi_{+} \partial_{M} \delta \Phi_{-}\right),
$$

where $d S^{M}$ is the volume element of a codimension-one, spacelike hypersurface $\mathcal{S}$, and $\delta \Phi_{ \pm}$ are the positive- and negative-frequency parts of the solution. In partcular, for a fixed-time slice $^{4}$ in the case at hand, $d S^{M}=\sqrt{\hat{g}} \hat{g}^{0 M} d x^{1} d x^{2} d x^{3} d^{d-4} y$. It follows then easily that the norm factorizes,

$$
\|\delta \Phi\|^{2}=\|\phi\|_{4}^{2} \times\|\psi\|^{2} \quad \text { where } \quad\|\psi\|^{2} \equiv \int d^{d-4} y \sqrt{[\hat{g}]} e^{2 A}|\psi|^{2}
$$

and $\|\phi\|_{4}^{2}$ denotes the norm of $\phi(x)$ in the unit-radius spacetime $\overline{\mathcal{M}}_{4}$. The non-trivial feature of the above expression is the warp factor inside the $y$-integral. Replacing $\delta \Phi$ by $\delta g_{\mu \nu}$ introduces two extra inverse metric factors, needed for the contraction of free indices. The extra warp factors cancel precisely those in $\delta g_{\mu \nu}$, so that again

$$
\|\delta g\|^{2}=\|h\|_{4}^{2} \times\|\psi\|^{2}
$$

where $\|h\|_{4}^{2}$ is the norm of the spin-2 perturbation $h_{\mu \nu}^{[\mathrm{tt}]}(x)$ in $\overline{\mathcal{M}}_{4}$. We conclude that the normalizable excitations are those for which the integral in (2.25) is finite. When expressed in terms of the wavefunction of the analog Schrödinger problem (2.21), the norm takes the expected canonical form

$$
\|\psi\|^{2}=\int d^{d-4} y \sqrt{[\bar{g}]}|\Psi|^{2}
$$

One immediate corollary is that $m^{2}$ is non-negative. This follows from the form of the mass-squared operator (2.20) and from the above expression for the norm:

$$
m^{2}\|\psi\|^{2}=-\int d^{d-4} y \psi\left(\partial_{a} \sqrt{[\hat{g}]} \hat{g}^{a b} e^{4 A} \partial_{b}\right) \psi=\int d^{d-4} y \sqrt{[\hat{g}]} e^{4 A}|\partial \psi|^{2} \geq 0
$$

where $\psi$ is here an eigenfunction with eigenvalue $m^{2}$. The mild assumption in the second step is that, upon integrating by parts, one picks up no contributions either from

\footnotetext{
${ }^{4}$ Strictly-speaking this argument applies only to backgrounds with a time-like Killing vector, either defined globally or at least in an asymptotic region where the notion of particles makes sense.
} 
singularities or from asymptotic regions. Conversely, since unitarity of the $\mathrm{SO}(2,3)$ spin-2 representation requires $m^{2} \geq 0$, a singularity that leads to a violation of this bound must correspond to a physically-unacceptable solution.

Another simple consequence, modulo the same mild assumption as above, is that a massless graviton must necessarily have $\psi(y)=$ constant [in which case the metric perturbation is proportional to the warp factor]. From the normalizability condition (2.25) we therefore conclude that:

$$
\text { the existence of a massless graviton } \Longleftrightarrow \int d^{d-4} y \sqrt{[\hat{g}]} e^{2 A}<\infty .
$$

This condition is, of course, automatically satisfied for compact internal spaces with smooth warp factor. It is, however, also a priori compatible with an infinite-volume internal space, as in the so-called Randall-Sundrum (RS) model II [2]. "Infinity" in this model is, nevertheless, an apparent horizon $[49,50]$ and the definition of the quantum theory requires a choice of boundary conditions. ${ }^{5}$ In the Karch-Randall model on the other hand, the geometry is geodesically-complete with $\mathrm{AdS}_{5}$ boundary regions. Since the integral in (2.29) diverges at the boundary of $\mathrm{AdS}_{5}$, there is no massless four-dimensional graviton in this model. This is also the case for the smooth interface geometries to which we turn now our attention.

\section{Interface solutions and their charges}

In this section we review the supersymmetric solutions of type IIB supergravity discovered in references $[19,20]$. We make a choice of coordinates most convenient for our purposes here, and relate the free parameters of the solutions to the charges of the underlying brane configuration and to the asymptotic values of the dilaton field. For the reader's convenience, we summarized in appendix A the field equations and Bianchi identities of the type-IIB bosonic fields, and their duality transformations.

\subsection{Local solutions: general form}

The solutions of interest are fibrations of $\mathrm{AdS}_{4} \times \mathrm{S}^{2} \times \mathrm{S}^{2}$ over a base space which is a Riemann surface $\Sigma$ with the topology of a disk. The general discussion of these solutions [20] is most convenient with a choice of complex coordinate that varies over the upper-half plane, but

\footnotetext{
${ }^{5}$ This problem is generic for all flat-brane geometries with a non-compact transverse space. Consider indeed an infinite transverse dimension parametrized by a flat coordinate $y$, and let $e^{A}$ vanish at infinity. For a particle moving along $y$ one has $e^{2 A}=C \sqrt{e^{2 A}-\dot{y}^{2}}$ for some constant $C$. Out at infinity $\dot{y} \simeq e^{A} \rightarrow 0$, so that the proper time integral reads:$$
\int d \tau=\int d t C^{-1} e^{2 A} \simeq C^{-1} \int d y e^{A} .
$$

Geodesic completeness of the spacetime requires that this integral diverge, so that the would-be horizon cannot be reached in finite proper time. The existence of a massless graviton requires on the other hand that $\int d y e^{2 A}$ be finite. These two conditions imply that at infinity $A \simeq-\nu \log y$ with $1>\nu>1 / 2$. Such asymptotic behavior is, however, ruled out by the weak-energy condition which requires that $A^{\prime \prime} \leq 0$ (the "holographic $c$-theorem) $[51,52]$. It can be checked that more than one infinite dimensions cannot help.
} 
for our purposes here we prefer to use a coordinate that varies over the infinite strip:

$$
\Sigma \equiv\left\{z \in \mathbb{C} \mid 0 \leq \operatorname{Im} z \leq \frac{\pi}{2}\right\} .
$$

The isometry $\mathrm{SO}(2,3) \times \mathrm{SO}(3) \times \mathrm{SO}(3)$ of the fibers is a symmetry of the solutions, which also preserve 16 super(conformal) symmetries. The solutions considered here will be completely specified by two functions $h_{1}(z, \bar{z})$ and $h_{2}(z, \bar{z})$ which are real harmonic and regular inside $\Sigma$, and which obey the boundary conditions (here $\partial_{\perp}$ is the normal derivative):

$$
h_{1}=\partial_{\perp} h_{2}=0 \quad \text { for } \quad \operatorname{Im} z=0, \quad h_{2}=\partial_{\perp} h_{1}=0 \quad \text { for } \quad \operatorname{Im} z=\frac{\pi}{2} .
$$

In writing down the solutions one also needs the dual harmonic functions, which are defined up to a constant by the following relations

$$
\begin{array}{rll}
h_{1}=-i\left(\mathcal{A}_{1}-\overline{\mathcal{A}}_{1}\right) & \rightarrow & h_{1}^{D}=\mathcal{A}_{1}+\overline{\mathcal{A}}_{1}, \\
h_{2}=\mathcal{A}_{2}+\overline{\mathcal{A}}_{2} & \rightarrow & h_{2}^{D}=i\left(\mathcal{A}_{2}-\overline{\mathcal{A}}_{2}\right) .
\end{array}
$$

Note that this ambiguity has a physical interpretation, namely constant shifts of $h_{1}^{D}$ and $h_{2}^{D}$ correspond, respectively, to gauge transformations of the RR and NSNS two-form gauge potentials. It is, furthermore, convenient to define the following combinations of $h_{1}, h_{2}$, and of their first derivatives (here $\partial=\partial / \partial z, \bar{\partial}=\partial / \partial \bar{z}$ ):

$$
\begin{aligned}
W & =\partial h_{1} \bar{\partial} h_{2}+\bar{\partial} h_{1} \partial h_{2}=\partial \bar{\partial}\left(h_{1} h_{2}\right), \\
N_{1} & =2 h_{1} h_{2}\left|\partial h_{1}\right|^{2}-h_{1}^{2} W \\
N_{2} & =2 h_{1} h_{2}\left|\partial h_{2}\right|^{2}-h_{2}^{2} W .
\end{aligned}
$$

In the conventions of appendix A, the supersymmetric solutions of refs. [19, 20] are then described by the following backgrounds:

$$
\text { Metric : } \quad d s^{2}=f_{4}^{2} d s_{\mathrm{AdS}_{4}}^{2}+f_{1}^{2} d s_{\mathrm{S}_{1}^{2}}^{2}+f_{2}^{2} d s_{\mathrm{S}_{2}^{2}}^{2}+4 \rho^{2} d z d \bar{z},
$$

where

$$
\begin{aligned}
f_{4}^{8} & =16 \frac{N_{1} N_{2}}{W^{2}}, & \rho^{8} & =\frac{N_{1} N_{2} W^{2}}{h_{1}^{4} h_{2}^{4}}, \\
f_{1}^{8} & =16 h_{1}^{8} \frac{N_{2} W^{2}}{N_{1}^{3}}, & f_{2}^{8} & =16 h_{2}^{8} \frac{N_{1} W^{2}}{N_{2}^{3}},
\end{aligned}
$$

and the $\mathrm{AdS}_{4}$ and 2-sphere metrics are normalized to unit radius;

$$
\begin{aligned}
\text { Dilaton : } & e^{4 \phi}=\frac{N_{2}}{N_{1}} ; \\
\text { 3-forms : } & F_{(3)}=\omega^{45} \wedge d b_{1}+i \omega^{67} \wedge d b_{2},
\end{aligned}
$$

where $\omega^{45}$ and $\omega^{67}$ are the volume forms of the unit-radius spheres $\mathrm{S}_{1}^{2}$ and $\mathrm{S}_{2}^{2}$, and

$$
\begin{aligned}
b_{1} & =2 i h_{1} \frac{h_{1} h_{2}\left(\partial h_{1} \bar{\partial} h_{2}-\bar{\partial} h_{1} \partial h_{2}\right)}{N_{1}}+2 h_{2}^{D}, \\
b_{2} & =2 i h_{2} \frac{h_{1} h_{2}\left(\partial h_{1} \bar{\partial} h_{2}-\bar{\partial} h_{1} \partial h_{2}\right)}{N_{2}}-2 h_{1}^{D} ; \\
\underline{5-\text { form }:} \quad F_{(5)} & =-4 f_{4}^{4} \omega^{0123} \wedge \mathcal{F}+4 f_{1}^{2} f_{2}^{2} \omega^{45} \wedge \omega^{67} \wedge\left(*_{2} \mathcal{F}\right),
\end{aligned}
$$


where $\omega^{0123}$ is the volume form of the unit-radius $\mathrm{AdS}_{4}, \mathcal{F}$ is a 1 -form on $\Sigma$ with the property that $f_{4}^{4} \mathcal{F}$ is closed, and $*_{2}$ denotes Poincaré duality with respect to the $\Sigma$ metric. The explicit expression for $\mathcal{F}$ is given by

$$
f_{4}^{4} \mathcal{F}=d j_{1} \quad \text { with } \quad j_{1}=3 \mathcal{C}+3 \overline{\mathcal{C}}-3 \mathcal{D}+i \frac{h_{1} h_{2}}{W}\left(\partial h_{1} \bar{\partial} h_{2}-\bar{\partial} h_{1} \partial h_{2}\right),
$$

where $\mathcal{C}$ is defined by the relation $\partial \mathcal{C}=\mathcal{A}_{1} \partial \mathcal{A}_{2}-\mathcal{A}_{2} \partial \mathcal{A}_{1}$ while $\mathcal{D}=\overline{\mathcal{A}}_{1} \mathcal{A}_{2}+\mathcal{A}_{1} \overline{\mathcal{A}}_{2}{ }^{6}$

The above set of expressions gives the local form of the general solution for the ansatz of refs. $[19,20]$. These expressions are invariant under conformal transformations of the coordinate $z$, which map, however, in general $\Sigma$ to a different disk-like domain of the complex plane. The following identities between the dilaton field and the metric factors will prove useful later:

$$
f_{1}^{2} f_{4}^{2}=4 e^{2 \phi} h_{1}^{2}, \quad f_{2}^{2} f_{4}^{2}=4 e^{-2 \phi} h_{2}^{2}, \quad \rho^{4} f_{1}^{2} f_{2}^{2}=4 W^{2} .
$$

Note that the general solutions consistent with the above isometries can also have a nonvanishing Ramond-Ramond scalar field. As has been shown however in [19], it is always possible to set the $\mathrm{RR}$ scalar to zero by a $\mathrm{SL}(2, \mathbb{R})$ duality rotation. In fact, since quantum effects break this symmetry to its discrete subgroup $\mathrm{SL}(2, \mathbb{Z})$, continuous rotations give a priori physically-inequivalent solutions. Since however only the metric enters in the analysis of the spin-2 spectrum, we wont need to keep track of this issue in the present work.

\section{2 $\quad \mathrm{AdS}_{5} \times \mathrm{S}^{5}$ and supersymmetric Janus}

Although any pair of harmonic functions $h_{1}, h_{2}$ gives a local solution of the supergravity equations, global consistency imposes stringent restrictions. As discussed in reference [20], for the solutions of interest the dilaton and all metric factors must be regular functions in the interior of $\Sigma$, but they may diverge at isolated points of the boundary. A further requirement is that the $\mathrm{AdS}_{4}$ scale factor, $f_{4}$, be everywhere non-vanishing, and that (with the exception of isolated points) the strip boundary, $\partial \Sigma$, should correspond to interior points of the ten-dimensional geometry.

Removing the singular points of $f_{4}$ separates $\partial \Sigma$ into a collection of boundary segments. The last regularity condition then implies that on each segment one of the two sphere radii must vanish. This is guaranteed by (3.1), as can be checked with the help of (3.11). To avoid a conical singularity, we need however also to fix the rate at which the sphere radii vanish. Explicitly, for $\operatorname{Im} z=\epsilon$ and $\operatorname{Im} z=\pi / 2-\epsilon^{\prime}$ with $\epsilon, \epsilon^{\prime} \ll 1$, we must require that

$$
\frac{f_{1}^{2}}{4 \rho^{2}} \simeq \epsilon^{2} \quad \text { and } \quad \frac{f_{2}^{2}}{4 \rho^{2}} \simeq \epsilon^{\prime 2}
$$

Further conditions may still be needed in order to constrain the allowed singularities at isolated points on the boundary of $\Sigma$. Admissible singularities include those that can be interpreted as coming from known string-theory branes.

\footnotetext{
${ }^{6}$ Note that, to match standard conventions for $\tilde{F}_{(5)}$, we have introduced an additional factor of 4 compared to $[19,20]$. Note also that the expressions (9.61) and (9.63) in [19] are missing the factor of $\mathcal{D}$.
} 
A simple choice for the harmonic functions, consistent with all the above physical requirements, is as follows:

$$
h_{1}=-i \alpha_{1} \sinh z+\text { c.c. } \quad \text { and } \quad h_{2}=\alpha_{2} \cosh z+\text { c.c. },
$$

where $\alpha_{1}$ and $\alpha_{2}$ are arbitrary real parameters. After a little algebra ${ }^{7}$ these give:

$$
\begin{array}{ll}
\rho^{4}=\left|\alpha_{1} \alpha_{2}\right|, & f_{4}^{2}=4 \rho^{2} \cosh ^{2}\left(\frac{z+\bar{z}}{2}\right), \\
f_{1}^{2}=4 \rho^{2} \sin ^{2}\left(\frac{z-\bar{z}}{2 i}\right), & f_{2}^{2}=4 \rho^{2} \cos ^{2}\left(\frac{z-\bar{z}}{2 i}\right) .
\end{array}
$$

Writing $z=x+i y$, one recognizes immediately the $\mathrm{AdS}_{5} \times \mathrm{S}^{5}$ metric,

$$
d s^{2}=L^{2}\left[d x^{2}+\cosh ^{2}(x) d s_{\mathrm{AdS}_{4}}^{2}+d y^{2}+\sin ^{2}(y) d s_{\mathrm{S}_{1}^{2}}^{2}+\cos ^{2}(y) d s_{\mathrm{S}_{2}^{2}}^{2}\right]
$$

with radius $L^{2}=4\left|\alpha_{1} \alpha_{2}\right|^{1 / 2}$. The choice (3.13) leads furthermore to vanishing 3-form fluxes, and to the following constant dilaton and 5-form backgrounds:

$$
e^{2 \phi}=\left|\frac{\alpha_{2}}{\alpha_{1}}\right| \quad \text { and } \quad F_{(5)}=-4 L^{4}(1+*) \omega^{4567 y},
$$

where $\omega^{4567 y}$ is the volume form on the unit $\mathrm{S}^{5}$, with orientation fixed by the sign of $\alpha_{1} \alpha_{2}$. The radius of the 5 -sphere is related as usual to the total $D 3$-brane charge:

$$
\int_{S^{5}} F_{(5)}=-4 \operatorname{Vol}\left(S^{5}\right)=-4 \pi^{3} L^{4}
$$

All the solutions that we will discuss will be obtained by deforming the harmonic functions of this basic $\mathrm{AdS}_{5} \times \mathrm{S}^{5}$ solution.

A one-parameter family of deformations, the supersymmetric Janus configurations [19], is obtained by the following trick: one notes that a translation along the strip preserves the boundary conditions of the harmonic functions. A common translation of the arguments of $h_{1}$ and $h_{2}$ is of course just a reparametrization. New regular solutions can, however, be found by a relative translation, i.e.

$$
h_{1}=-i \alpha_{1} \sinh \left(z-\frac{\Delta \phi}{2}\right)+\text { c.c. } \quad \text { and } \quad h_{2}=\alpha_{2} \cosh \left(z+\frac{\Delta \phi}{2}\right)+\text { c.c. },
$$

where $\Delta \phi$ is a real parameter (whose name will be justified in a moment). The regularity of the Janus solution has been established in ref. [19]. In the asymptotic regions $x \rightarrow \pm \infty$ we expect it to approach the basic $\mathrm{AdS}_{5} \times \mathrm{S}^{5}$ geometry. To find the asymptotic values of the radius and dilaton requires some care, because $W$ vanishes at the leading exponential order. A simple calculation actually gives

$$
W=-\alpha_{1} \alpha_{2} \cosh (\Delta \phi) \sin (2 y),
$$

\footnotetext{
${ }^{7}$ The following identities are useful: $(c+\bar{c})(s-\bar{s})=(c \bar{s}-\bar{c} s)(1+c \bar{c}+s \bar{s}),(c+\bar{c})(c \bar{s}-\bar{c} s)=(s-\bar{s})(1+c \bar{c}-s \bar{s})$ and $(s-\bar{s})(c \bar{s}-\bar{c} s)=(c+\bar{c})(1-c \bar{c}+s \bar{s})$, where $c \equiv \cosh z, s \equiv \sinh z$ and bars are complex conjugates.
} 
so the deformation simply rescales $W$ by a factor $\cosh (\Delta \phi)$. Using this fact, and keeping the leading exponential behavior for all other terms in the expressions (3.3) to (3.6), leads to the following values for the dilaton and the radius of the two asymptotic $\operatorname{AdS}_{5} \times \mathrm{S}^{5}$ regions, at $x \rightarrow \pm \infty$ :

$$
L^{4}=16\left|\alpha_{1} \alpha_{2}\right| \cosh (\Delta \phi) \quad \text { and } \quad e^{2 \phi_{ \pm}}=\left|\frac{\alpha_{2}}{\alpha_{1}}\right| e^{ \pm \Delta \phi} .
$$

The Janus configuration describes a smooth supersymmetric domain wall, across which the dilaton changes by a total amount $\Delta \phi$. The wall lives in the near-horizon geometry of D3-branes. This simplest solution has no five-brane charge, as is obvious from the fact that there is no non-contractible 3 -cycle that can support it.

\subsection{Non-vanishing five-brane charge}

Adding five-brane charge to the supersymmetric Janus solution requires harmonic functions with singularities at the boundaries of the strip. These singularities describe new asymptotic regions of the ten-dimensional geometry. ${ }^{8}$ Proceeding in steps, we consider first the following two-parameter deformation of Janus:

$$
\begin{aligned}
& h_{1}=-i \alpha_{1} \sinh \left(z-\beta_{1}\right)+\text { c.c. } \\
& h_{2}=\left[\alpha_{2} \cosh \left(z-\beta_{2}\right)-\gamma \ln \left(\tanh \frac{z}{2}\right)\right]+\text { c.c. } .
\end{aligned}
$$

All the parameters in these expressions must be real. The function $\ln \left(\tanh \frac{z}{2}\right)$ is purely imaginary for $\operatorname{Im} z=\frac{\pi}{2}$, so $h_{2}$ continues to vanish on the upper boundary of the strip. It also obeys a Neumann condition on the lower boundary, except at the origin, $z=0$, where it has a logarithmic singularity. This is not very surprising, since we expect the metric and the dilaton to be singular at the position of a 5-brane.

To show why the above solution has NS5-brane charge, we need to identify a noncontractible 3 -cycle that can support 3 -form flux. Consider an open curve, $\mathcal{I}$, starting and ending on the lower boundary of the infinite strip. Since $S_{1}^{2}$ shrinks to zero size at both ends, $\mathcal{I}$ and this 2 -sphere fiber gives a 3 -cycle with the topology of a 3 -sphere. When $\gamma=0$ this cycle is contractible, because $\mathcal{I}$ can shrink continuously to a point. For $\gamma \neq 0$ on the other hand, a curve with its endpoints on either side of $z=0$ cannot be contracted. There is no RR 3-form flux through this cycle because, as can be seen from eq. (3.7), the RR 3 -form is proportional to the volume of the second sphere, $\mathrm{S}_{2}^{2} \cdot{ }^{9}$ The NS-NS flux through

\footnotetext{
${ }^{8}$ As explained in reference [20] each five-brane singularity can be split into a pair of singularities of branch-cut type like those describing the asymptotic $\operatorname{AdS}_{5} \times \mathrm{S}^{5}$ regions. Note that after such a splitting, there may appear conical singularities in the bulk of $\Sigma$. To see this, note that in the upper-half-plane coordinates the function $W$ and correspondingly the $\Sigma$-metric, $\rho$, vanish at isolated points in the bulk of $\Sigma$. It has not been explicitly checked whether these points lead to conical singularities or not.

${ }^{9}$ The reader can check that the integral of $\operatorname{Im} F_{(3)}$ over $S_{2}^{2} \times \mathcal{I}$ does not vanish, so the solution (3.21) has a non-zero RR 3-form field. But $S_{2}^{2} \times \mathcal{I}$ is a cycle with boundaries and, in accordance with the absence of D5-brane charge, the integral of $\operatorname{Im} F_{(3)}$ over any closed 3-cycle is zero.
} 
$\mathrm{S}_{1}^{2} \times \mathcal{I}$, on the other hand, reads

$$
Q_{\mathrm{NS} 5}=\int_{\mathcal{I} \times S_{1}^{2}} \operatorname{Re} F_{(3)}=\int_{\mathcal{I}} d b_{1} \int_{S_{1}^{2}} \omega^{45}=\left.4 \pi b_{1}\right|_{\partial \mathcal{I}}=\left.8 \pi h_{2}^{D}\right|_{\partial \mathcal{I}}
$$

where $\partial \mathcal{I}$ is the boundary of $\mathcal{I}$. The last of the above equalities follows from eq. (3.8), and the vanishing of $h_{1}$ on the real- $z$ axis. The function dual to $h_{2}$ is

$$
h_{2}^{D}=\left[i \alpha_{2} \cosh \left(z-\beta_{2}\right)-i \gamma \ln \left(\tanh \frac{z}{2}\right)\right]+\text { c.c. },
$$

from which it follows easily that $\left.h_{2}^{D}\right|_{\partial \mathcal{C}}=2 \pi \gamma$. Note that the entire contribution to the charge comes from the imaginary part of the logarithm, and only depends on the topological class of the open curve $\mathcal{I}$, i.e. on whether its two endpoints lie on the same or on opposite sides of $z=0$. Inserting in (3.22) gives the relation between $\gamma$ and the NS5-brane charge,

$$
Q_{\mathrm{NS} 5}=16 \pi^{2} \gamma
$$

To relate the remaining four parameters of (3.21) to physical parameters of the solution, we consider again the asymptotic geometry in the two limits $x \rightarrow \pm \infty$. Note first that the extra term in the expression for $h_{2}$ has the asymptotic behavior

$$
-\gamma \ln \left(\tanh \frac{z}{2}\right) \simeq \begin{cases}2 \gamma e^{-z} & \text { when } x \rightarrow \infty, \\ -i \gamma \pi+2 \gamma e^{z} & \text { when } x \rightarrow-\infty .\end{cases}
$$

Adding this to the hyperbolic cosine gives

$$
h_{2} \simeq \alpha_{2}^{ \pm} \cosh \left(z-\beta_{2}^{ \pm}\right)+\text {c.c. } \quad \text { when } x \rightarrow \pm \infty,
$$

where

$$
\alpha_{2}^{ \pm}=\alpha_{2} \sqrt{1+\frac{4 \gamma}{\alpha_{2}} e^{\mp \beta_{2}}}, \quad e^{\beta_{2}^{ \pm}}=e^{\beta_{2}}\left(1+\frac{4 \gamma}{\alpha_{2}} e^{\mp \beta_{2}}\right)^{ \pm 1 / 2}
$$

By our previous argument, we may now compute the radius and the value of the dilaton in the two asymptotic $\mathrm{AdS}_{5} \times \mathrm{S}^{5}$ regions with the result:

$$
L_{ \pm}^{4}=\frac{Q_{\mathrm{D} 3}^{ \pm}}{2 \pi^{3}}=16\left|\alpha_{1} \alpha_{2}^{ \pm}\right| \cosh \left(\beta_{1}-\beta_{2}^{ \pm}\right) \quad \text { and } \quad e^{2 \phi_{ \pm}}=\left|\frac{\alpha_{2}^{ \pm}}{\alpha_{1}}\right| e^{ \pm\left(\beta_{1}-\beta_{2}^{ \pm}\right)} .
$$

Notice that the asymptotic radii and D3-branes charges need not be the same at the two ends, as was the case in the Janus solution. Indeed, the D3-branes can either intersect or end on the NS5-brane stack, which is allowed to carry D3-brane charge [53]. The fraction of D3-brane charge dissolved inside the NS5-branes is

$$
\frac{Q_{\mathrm{D} 3}^{-}-Q_{\mathrm{D} 3}^{+}}{Q_{\mathrm{D} 3}^{+}+Q_{\mathrm{D} 3}^{-}}=\frac{2 \gamma \sinh \beta_{1}}{2 \gamma \cosh \beta_{1}+\alpha_{2} \cosh \left(\beta_{1}-\beta_{2}\right)} .
$$

This vanishes when $\gamma=0$, since in this case there are no NS5-branes, but also when $\beta_{1}=0$ which corresponds to all D3-branes intersecting the NS5-branes. The other extreme, where 
this ratio approaches one, corresponds either to $\gamma \rightarrow \infty$, or to $\beta_{1}, \beta_{2} \rightarrow \infty$ with the difference $\beta_{1}-\beta_{2}$ held fixed. In these limits the NS5-brane stack absorbs the totality of D3-brane charge in its worldvolume.

A solution with D5-brane charge can be obtained from eq. (3.21) by an S-duality transformation. The harmonic functions transform as a doublet of $\operatorname{SL}(2, \mathbb{Z})$, so that under this transformation $h_{1} \rightarrow h_{2}$ and $h_{2} \rightarrow-h_{1}$. The transformation also exchanges the two 2-spheres. To restore the initial boundary conditions (3.1), we furthermore make the (analytic) reparametrization of the strip $z \rightarrow i \pi / 2-z$. The D5-brane background corresponds thus, finally, to the functions

$$
\begin{aligned}
& h_{1}=\left[i \alpha_{2} \sinh \left(z+\beta_{2}\right)+\gamma \ln \left(\tanh \left(\frac{i \pi}{4}-\frac{z}{2}\right)\right)\right]+\text { c.c. } \\
& h_{2}=\alpha_{1} \cosh \left(z+\beta_{1}\right)+\text { c.c. }
\end{aligned}
$$

It can be easily checked that the metric of this solution is the same as that of the NS5 brane, while the sign of the dilaton is flipped, and the NSNS and RR 3-forms are exchanged. This is precisely the action of the S-duality element of $\mathrm{SL}(2, \mathbb{Z})$.

It is now easy to write down a solution, which involves both NS5-brane and D5-brane stacks wrapping the two different 2 -spheres of the background geometry. The corresponding harmonic functions read:

$$
\begin{aligned}
& h_{1}=\left[-i \alpha_{1} \sinh \left(z-\beta_{1}\right)-\gamma_{1} \ln \left(\tanh \left(\frac{i \pi}{4}-\frac{z-\delta_{1}}{2}\right)\right)\right]+\text { c.c. }, \\
& h_{2}=\left[\alpha_{2} \cosh \left(z-\beta_{2}\right)-\gamma_{2} \ln \left(\tanh \left(\frac{z-\delta_{2}}{2}\right)\right)\right]+\text { c.c. }
\end{aligned}
$$

This solution describes the near-horizon geometry of stacks of intersecting D3-branes, NS5branes and D5-branes, whose worldvolumes in the asymptotically-flat spacetime are along the directions (0123), (012456) and (012789). The five-brane charges are

$$
Q_{\mathrm{D} 5}=16 \pi^{2} \gamma_{1}, \quad Q_{\mathrm{NS} 5}=16 \pi^{2} \gamma_{2} .
$$

The asymptotic values of the $\mathrm{AdS}_{5} \times \mathrm{S}^{5}$ radii and the dilaton field are:

$$
L_{ \pm}^{4}=16\left|\alpha_{1}^{ \pm} \alpha_{2}^{ \pm}\right| \cosh \left(\beta_{1}^{ \pm}-\beta_{2}^{ \pm}\right) \quad \text { and } \quad e^{2 \phi_{ \pm}}=\left|\frac{\alpha_{2}^{ \pm}}{\alpha_{1}^{ \pm}}\right| e^{ \pm\left(\beta_{1}^{ \pm}-\beta_{2}^{ \pm}\right)},
$$

where $\alpha_{j}^{ \pm}$and $\beta_{j}^{ \pm}$for $j=1,2$ are given by:

$$
\alpha_{j}^{ \pm}=\alpha_{j} \sqrt{1+\frac{4 \gamma_{j}}{\alpha_{j}} e^{ \pm\left(\delta_{j}-\beta_{j}\right)}}, \quad e^{\beta_{j}^{ \pm}}=e^{\beta_{j}}\left(1+\frac{4 \gamma_{j}}{\alpha_{j}} e^{ \pm\left(\delta_{j}-\beta_{j}\right)}\right)^{ \pm 1 / 2}
$$

The ratio $L_{-}^{4} / L_{+}^{4}$ determines, as previously, the fraction of D3-branes dissolved into the two stacks of 5-branes. The fractions dissolved separately into the D5-brane stack, and the NS5 stack, can be computed from the integrals of the RR 5-form over $\mathcal{I} \times S_{1}^{2} \times S_{2}^{2}$, where $\mathcal{I}$ is a semicircle around the corresponding logarithmic singularity on the strip boundary. 
There is, actually, a well-known subtlety in the definition of D3-brane charge due to the Chern-Simons term of the type-IIB action, as reviewed nicely in reference [54]. The gauge-invariant and conserved Maxwell charge differs in general from the Page charge which (being quantized and localized) is the one that counts the number of D3-branes. In the asymptotic $\mathrm{AdS}_{5}$ regions the two notions of charge coincide, so that their difference, i.e. the total number of D3-branes dissolved in the five-brane stacks, is unambiguous. The separate fractions of D3-branes going into the NS5-stack and the D5-stack may suffer from a gauge ambiguity, but we will not need their explicit expressions here. We just note that the total parameter count is correct: there are seven physical parameters, the two asymptotic values of the dilaton, the two 5-brane charges, and the three D3-brane charges, i.e. the numbers of D3-branes that are (i) dissolved in the NS5-branes, (ii) dissolved in the D5-branes, or (iii) intersecting both stacks. This is the same as the number of parameters in (3.31), if one excludes the spurious parameter that fixes the origin of the real- $z$ axis.

The solution (3.31) can be generalized further by including any number of five-brane singularities, both in the upper and in the lower boundaries of the strip. These describe configurations with different fractions of D3-branes dissolved into more than two distinct fivebrane stacks. They can be analyzed by a straightforward extension of the above discussion.

\section{Localized gravity: what to look for?}

In this section we briefly review the Karch-Randall model [17], and in particular those features of its prototype thin-brane geometry that are responsible for the localization of gravity. A key qualitative feature of this geometry is that the warp factor has a local bump, whose height generates a large scale hierarchy. As we will see, the Janus configuration does not share this qualitative feature, and it cannot therefore localize gravity. The reason is that a dilaton domain wall has no a priori prescribed tension and thickness, because its superpotential vanishes. Solutions with both NS5- and D5-brane charge are, in this regard, more promising, but their detailed analysis will be postponed to a forthcoming companion paper [41].

\subsection{The Karch-Randall model}

The starting point of the Karch-Randall model is the effective 5D thin-brane action

$$
I_{\mathrm{KR}}=-\frac{1}{2 \kappa_{5}^{2}} \int d^{4} x d y \sqrt{g}\left(R+\frac{12}{L^{2}}\right)+\lambda \int d^{4} x \sqrt{[g]_{4}},
$$

where $[g]_{4}$ is the determinant of the induced metric at $y=0$. This action depends on three dimensionful parameters: the $5 \mathrm{D}$ gravitational coupling $\kappa_{5}$, the tension $\lambda$ of the thin 3 -brane, and the radius of curvature $L$ of the bulk spacetime. The important dimensionless combination is $\kappa_{5}^{2} \lambda L$, or equivalently the length parameter

$$
y_{0}=L \operatorname{arctanh}\left(\frac{\kappa_{5}^{2} \lambda L}{6}\right)
$$




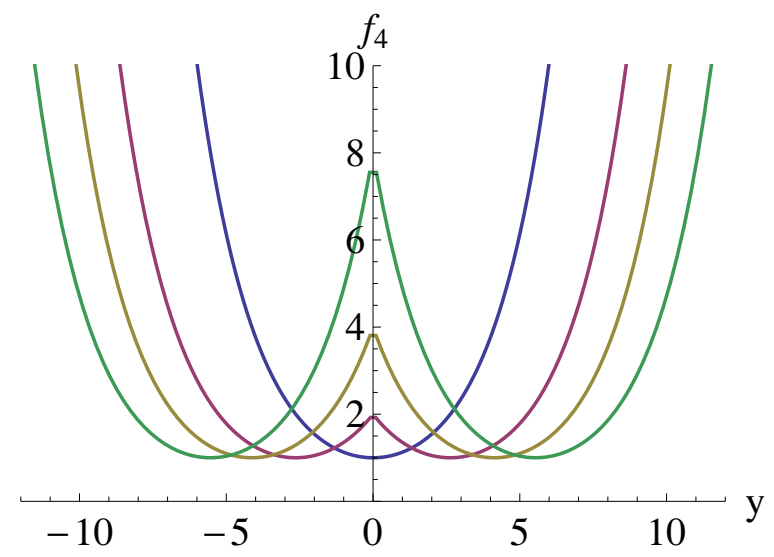

Figure 1. The $\mathrm{AdS}_{4}$ warp factor $\left(f_{4}\right)$ of the Karch-Randall geometry with $L=1$ for $l=1,2,4$ and 8 (blue, purple, yellow, and green, respectively).

in terms of which the solution of the Einstein equations can be written as $[49,55,56]$

$$
d s^{2}=L^{2} \cosh ^{2}\left(\frac{y_{0}-|y|}{L}\right) \bar{g}_{\mu \nu} d x^{\mu} d x^{\nu}+d y^{2}
$$

with $\bar{g}_{\mu \nu}$ the metric of the unit-radius $\mathrm{AdS}_{4}$. The spacetime (4.3) consists of two identical slices of $\mathrm{AdS}_{5}$ of radius $L$, glued by Israel matching conditions at the location of the thin 3 -brane. The parameter $y_{0}$ is the turn-around point of the warp factor, and it moves to infinity as one tunes $\kappa_{5}^{2} \lambda L \rightarrow 6$ (see figure 1 ).

The physical parameters in four dimensions are the radius of curvature $\ell$ of the $\mathrm{AdS}_{4}$ brane-world, Newton's constant $G_{N}$, and the characteristic Kaluza-Klein length, $\ell_{\mathrm{KK}}$, below which Newton's force law is modified by 5D physics. Overlooking the fact that our actual universe is de Sitter, not anti-de Sitter, we have the following observational requirements (for short-range tests of gravity see e.g. ref. [57, 58]):

$$
\sqrt{\ell \ell_{\text {Planck }}} \sim 1 \mathrm{~mm}, \quad \frac{\ell}{\ell_{\text {Planck }}} \sim 10^{62}, \quad \text { and } \ell_{\mathrm{KK}}<1 \mathrm{~mm} .
$$

To convert these to constraints on the $5 \mathrm{D}$ parameters, one first notes that

$$
\ell^{2}=e^{2 A(0)}=L^{2} \cosh ^{2}\left(\frac{y_{0}}{L}\right)
$$

so for the brane-world to be almost flat we need $y_{0}$ bigger (but not hierarchically bigger) than $L$. The geometry near the 3 -brane can then be approximated by the exponential warp factor of the Randall-Sundrum model [2],

$$
d s^{2} \simeq \ell^{2} e^{-2|y| / L} \bar{g}_{\mu \nu} d x^{\mu} d x^{\nu}+d y^{2} .
$$

The role of the geometry beyond the turn-around points $y_{0}$ is essentially to replace the adhoc boundary condition at the $\mathrm{AdS}_{5}$ horizon by a suitable normalizability condition. The main conclusions of reference [2] can then be carried over to this bent-brane case. The 
effective 4D Newton's constant, in particular, reads

$$
8 \pi G_{N}=\kappa_{5}^{2} e^{2 A(0)}\left|\psi_{0}(0)\right|^{2} \simeq \kappa_{5}^{2} e^{2 A(0)}\left(\int_{-y_{0}}^{y_{0}} d y e^{2 A}\right)^{-1} \simeq \kappa_{5}^{2} / L
$$

where $\psi_{0}(y)$ is the wavefunction of the lightest, nearly-massless graviton mode. The corrections to Newton's law from the exchange of higher excitations are given by

$$
V_{\text {Newton }}+\Delta V \simeq-\frac{G_{N} m_{1} m_{2}}{r}\left(1+\gamma \frac{L^{2}}{r^{2}}+\cdots\right)
$$

with $\gamma$ a numerical constant. Note that the relation between $G_{N}$ and the 5D coupling is the same as for a standard Kaluza-Klein circle of size $L$, but the corrections to Newton's law in the two cases are different. Either way, in view of the experimental constraint (4.4) we need $L$ to be less than (a fraction of) the millimeter.

A key feature of the above prototype geometry is the existence of a local maximum of the warp factor near $y \simeq 0$. Its immediate vicinity supports the nearly-constant, nearlymassless mode $\psi_{0}$, whose mass has been shown in ref. [59] to be given by $m_{0}^{2} \simeq 3 L^{2} / 2 \ell^{2}$. This is of the same order as the blueshift between the local maximum at $y=0$ and the minima at $y= \pm y_{0}$. The estimate for the wavefunction in (4.7) is based on the assumption that $\psi_{0}(y)$ is almost constant at $y \ll y_{0}$, so that the contribution to its norm vanishes exponentially fast away from the brane.

The large blueshift between the points $y=0$ and $y=y_{0}$ is crucial for suppressing the contribution of the excited modes. These have $\mathrm{AdS}_{4}$ masses $m \sim 1$, so that they mediate interactions whose range is comparable to the radius of curvature, $\ell$, of the braneworld. Their support is, however, centered around $y_{0}$, and they have very small amplitude at the position $y=0$ of the 3 -brane. The actual calculation (see e.g. [2, 39, 60-63] and references therein) gives $|\psi(0 \mid m)|^{2} \sim m L^{2} / \ell^{2}$, so that the contribution to the gravitational force,

$$
\delta V=V_{\text {Newton }} \times\left|\psi_{0}(0)\right|^{-2} \sum_{\left\{m \neq m_{0}\right\}}|\psi(0 \mid m)|^{2} e^{-m r / \ell},
$$

takes, at distances $L \ll r \ll \ell$, the subleading form exhibited in equation (4.8). The precise form of the KK correction (which also includes contributions from non-TT modes) will, in general, depend on details of the geometry. What appears, however, to be essential for the localization of gravity is the very large suppression of the excited modes at the location of the brane.

Some further intuition on this issue can be derived from the equivalent Schödinger problem defined in eqs. (2.21) and (2.22). Using a coordinate in which the rescaled internal metric is constant $\left(\bar{g}_{55}=e^{-2 A} \hat{g}_{55}=\ell^{-2}\right)$ one finds

$$
-\frac{d^{2} \Psi}{d z^{2}}+\left[\frac{15}{4\left(L^{2}+z^{2}\right)}-\frac{3}{L} \delta(z)\right] \Psi \simeq \frac{m^{2}}{\ell^{2}} \Psi \quad \text { where } \quad 1+\frac{|z|}{L} \simeq e^{|y| / L} .
$$

These expressions are actually valid for $z \ll \ell$, while the exact potential turns around and diverges at some large but finite value $z_{0}$ [17]. The key feature of this analog potential is its volcano shape, which traps the nearly massless graviton near $z \sim 0$, and expells 
from this region the excited modes. In previous studies of thick-brane models $[38,56$, 64] this shape was engineered by appropriately tuning an adhoc superpotential of scalar fields. One important issue that concerns us here is whether such a shape can be obtained in string theory.

We complete this section with a comment on gravity localization as seen from the perspective of the dual defect field theories. As explained in ref. [32], the mode expansion of supergravity fields is mapped to the expansion of conformal operators in the $4 \mathrm{D}$ bulk, as they approach the three-dimensional defect. A nearly-massless localized $\mathrm{AdS}_{4}$ graviton should thus correspond to an operator $\mathcal{O}_{0}$ with dimension $\Delta \simeq 3$ in the expansion of the energy-momentum tensor. Furthermore, the behavior of the corrections to Newton's force in the Randall-Sundrum model corresponds to the $r^{-4}$ fall-off of the two-point function of the bulk energy-momentum tensor $[35,36] .{ }^{10}$ It is also interesting to note that, while the 3-point functions of the energy-momentum tensor in a homogeneous CFT are completely fixed by Ward identities, this is not the case for the 3-point functions involving the reduced operator $\mathcal{O}_{0}$. This reflects the fact that our hypothetical nearly-massless graviton need not have universal couplings to all the forms of matter. ${ }^{11}$

\subsection{Warp factors and a limiting geometry}

It has been argued in reference [18] that intersecting D3-branes and D5-branes could provide a string-theory embedding of the Karch-Randall model. The underlying hypothesis was that the back-reaction of the D5-branes on the $\mathrm{AdS}_{5} \times \mathrm{S}^{5}$ geometry could be well approximated by the effective thin-brane action (4.1). Given the explicit solutions of D'Hoker et al $[19,20]$ one can try to find out whether this assumption was justified. The questions to ask are: (1) does the warp factor develop a "local bump" with a large hierarchy of scales? and (2) can the KK modes, both on the strip and on the two-spheres, be kept within the experimental limits? The second question is of course harder to address, since it depends on the detailed shape of the analog potential as well as the metric $\bar{g}_{a b}$ in the internal space.

The warp factor in the solutions of D'Hoker et al reads

$$
e^{2 A}=f_{4}^{2}=2\left(\frac{N_{1} N_{2}}{W^{2}}\right)^{\frac{1}{4}},
$$

where $W, N_{1}, N_{2}$ have been defined in eq. (3.3). We first consider the case of Janus, for which the harmonic functions (3.18) can be written as

$$
h_{1}=2 \alpha_{1} \cosh \left(x-\frac{\Delta \phi}{2}\right) \sin y, \quad h_{2}=2 \alpha_{2} \cosh \left(x+\frac{\Delta \phi}{2}\right) \cos y,
$$

where $z=x+i y$. A simple calculation then gives

$$
h_{1} h_{2}=\alpha_{1} \alpha_{2} \sin 2 y(\cosh \Delta \phi+\cosh 2 x), \quad W=-\alpha_{1} \alpha_{2} \sin 2 y \cosh \Delta \phi,
$$

\footnotetext{
${ }^{10}$ To touch basis with the usual discussion in Poincaré coordinates, note that the distance $r$ between two points on the $\mathrm{AdS}_{4}$ brane is related to the geodesic distance $R$ in the embedding $\mathrm{AdS}_{5}$ spacetime through $L^{2}\left(\cosh ^{2} \frac{R}{L}-1\right)=\ell^{2}\left(\cosh ^{2} \frac{r}{\ell}-1\right)$. Removing the standard flat-brane cutoff in the AdS/CFT correspondence amounts to zooming into the region $L \ll r \ll \ell$.

${ }^{11}$ We thank J. Penedones and S. Rychkov for a discussion of this point.
} 

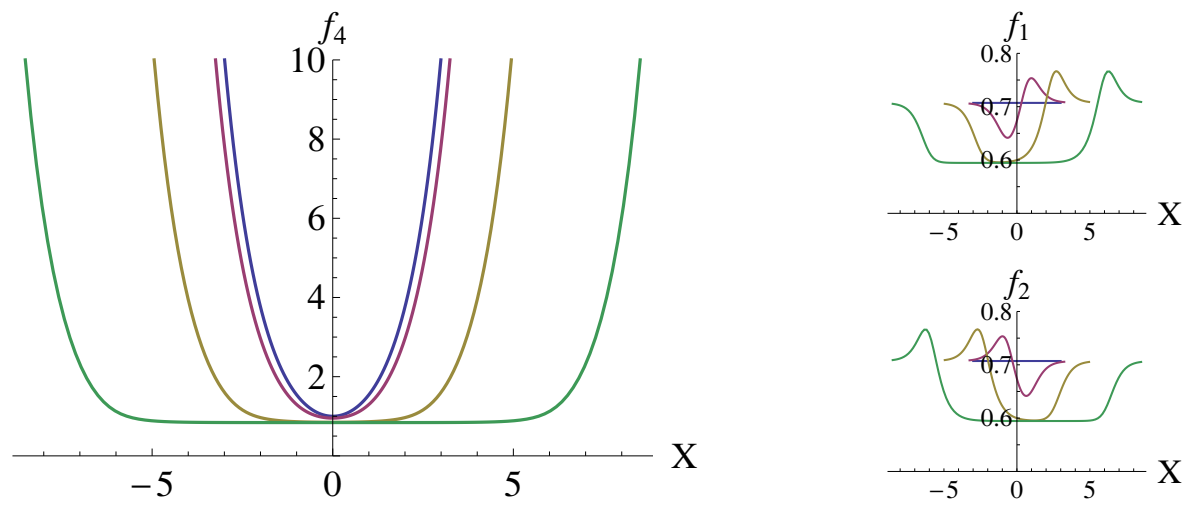

Figure 2. The $\mathrm{AdS}_{4}$ warp factor $\left(f_{4}\right)$ and the sphere radii $\left(f_{1}, f_{2}\right)$ of the Janus geometry for dilaton jumps $\Delta \phi=0,1,4$ and 10 (blue, purple, brown and green curves, respectively). The horizontal axis gives the invariant distance from the strip center, along the line $y=\pi / 4$. The asymptotic $\mathrm{AdS}_{5}$ radii have been set to one, and the dilaton in the left asymptotic region has been set to zero. Note the absence of a local maximum of the warp factor.

and after a slightly more tedious algebra:

$$
\begin{gathered}
N_{1}=\alpha_{1}^{3} \alpha_{2} \sin 2 y[\sinh \Delta \phi \cos 2 y \sinh (2 x+\Delta \phi)-\sinh \Delta \phi \sinh 2 x(\cosh 2 x+2 \cosh \Delta \phi) \\
\left.+\cosh \Delta \phi\left(1+\cosh ^{2} 2 x+2 \cosh 2 x \cosh \Delta \phi\right)\right], \\
N_{2}=\alpha_{1} \alpha_{2}^{3} \sin 2 y[\sinh \Delta \phi \cos 2 y \sinh (2 x-\Delta \phi)+\sinh \Delta \phi \sinh 2 x(\cosh 2 x+2 \cosh \Delta \phi) \\
\left.+\cosh \Delta \phi\left(1+\cosh ^{2} 2 x+2 \cosh 2 x \cosh \Delta \phi\right)\right] .
\end{gathered}
$$

As a check note that for $\Delta \phi=0$ the square brackets in the above expressions reduce to $\left[4 \cosh ^{4} x\right]$, so that the warp factor is $y$-independent. This is indeed the $\operatorname{AdS}_{5} \times \mathrm{S}^{5}$ limit in which the geometry factorizes.

For $\Delta \phi \neq 0$ the square brackets in (4.14) depend on the coordinate $y$, and the geometry does not factorize. Note in passing the symmetry under the reflection $(x, y) \rightarrow(-x, \pi / 2-$ $y$ ), which exchanges the harmonic functions $h_{1}$ and $h_{2}$ and thus leaves the metric invariant. Along the middle line of the strip, i.e. at $y=\pi / 4$, the wrap factor is given up to an overall constant by

$$
e^{8 A} \propto\left(1+\cosh ^{2} 2 x+2 \cosh 2 x \cosh \Delta \phi\right)^{2}-\tanh ^{2} \Delta \phi \sinh ^{2} 2 x(\cosh 2 x+2 \cosh \Delta \phi)^{2} .
$$

Figure 2 shows $e^{A} \equiv f_{4}$ as a function of the invariant distance $X=\int_{0}^{ \pm x} 2 \rho d x$, for different values of the parameter $\Delta \phi$. As these plots show, and as one can check explicitly from the above expression, $f_{4}$ is an even function of $x$ increasing monotonically from the center out to the $\mathrm{AdS}_{5}$ boundary regions. The absence of a local bump reminiscent of the thin-brane geometry of [17] is a clear indication that Janus cannot possibly localize gravity.

One other thing seen in figure 2 is that the $\mathrm{AdS}_{5}$ trap becomes broader and flatter as the dilaton jump $\Delta \phi$ is tuned up. Interestingly, the limit $\Delta \phi \rightarrow \infty$ leads to a smooth, 
geodesically-complete geometry. Indeed, for $|x| \ll \Delta \phi$ one gets

$$
\begin{aligned}
& N_{1} \simeq \frac{1}{4} \alpha_{1}^{3} \alpha_{2} e^{2 \Delta \phi} e^{-2 x} \sin 2 y\left(1+2 \sin ^{2} y\right), \\
& N_{2} \simeq \frac{1}{4} \alpha_{1} \alpha_{2}^{3} e^{2 \Delta \phi} e^{2 x} \sin 2 y\left(1+2 \cos ^{2} y\right),
\end{aligned}
$$

leading to the following expressions for the metric and the dilaton:

$$
\begin{aligned}
& d s^{2}= L^{2}\left(3+\sin ^{2} 2 y\right)^{1 / 4}\left[\frac{1}{2} d s_{\mathrm{AdS}_{4}}^{2}+d z d \bar{z}\right. \\
&\left.\quad+\left(\frac{\sin ^{2} y}{1+2 \sin ^{2} y}\right) d s_{\mathrm{S}_{1}^{2}}^{2}+\left(\frac{\cos ^{2} y}{1+2 \cos ^{2} y}\right) d s_{\mathrm{S}_{2}^{2}}^{2}\right] \\
& e^{2 \phi}=\left|\frac{\alpha_{2}}{\alpha_{1}}\right|\left(\frac{1+2 \cos ^{2} y}{1+2 \sin ^{2} y}\right)^{1 / 2} e^{2 x} .
\end{aligned}
$$

This limiting geometry describes therefore a five-dimensional, non-compact spacetime $\left(\mathrm{AdS}_{4} \times \mathbb{R}\right)$ with a dilaton varying linearly in $\mathbb{R}$, and a warp factor varying smoothly over a deformed 5 -sphere. For large but finite $\Delta \phi$ the nearly-flat fifth dimension has size $\sim \Delta \phi$, and the Kaluza-Klein excitations should therefore have characteristic spacings $\delta m \simeq(\Delta \phi)^{-1}$. This will be indeed confirmed by the numerical analysis of the Janus spectrum in section 6.2 .

The reason for this behavior of the Janus solution can be understood if one notes that the dilaton has no superpotential at the classical level. This should be contrasted with toy models of thick brane worlds $[38,56,64]$, where the superpotential can be engineered to produce the desired warp factor. The tension and thickness of scalar-field domain walls are, in particular, tunable parameters in these toy models. Dilaton domain walls, on the other hand, would tend to spread to infinite thickness and zero tension in the absence of gravity. They are only stabilized in the case at hand by the asymptotic $\mathrm{AdS}_{5}$ regions.

Five-branes are more promising in this respect, since they are stable in flat spacetime. Figure 3 depicts the $\mathrm{AdS}_{4}$ warp factor and the sphere radii for solutions with NS5-brane charge, while solutions with both NS5-brane and D5-brane charge are shown in figure 4. As can be seen from these figures, the warp factor does indeed exhibit a characteristic bump, but this grows wider and flatter as it gets taller. It thus looks as if a scale hierarchy cannot be arranged without decompactifying the internal space. This question will be analyzed in detail in ref. [41]. For now, we conclude with a simple observation: while the warp-factor bump is tiny in figure 3, it is much more pronounced in figure 4 . The reason is that the NS5-brane stack (whose tension in the Einstein frame scales as $e^{\phi / 2}$ ) pushes the dilaton field to a larger value, thereby reducing its back-reaction on the local geometry. The D5branes (whose tension scales like $e^{-\phi / 2}$ ) exerts an opposite pressure on the dilaton. When there are both NS5-branes and D5-branes the dilaton reaches an equilibrium value, and the total tension cannot be further reduced.

\section{Spectral problem on the strip}

We go back now to the eigenmode equation of section 2, and specialize it to the interface solutions of D'Hoker et al. We will see, in particular, that for excitations that are constant 

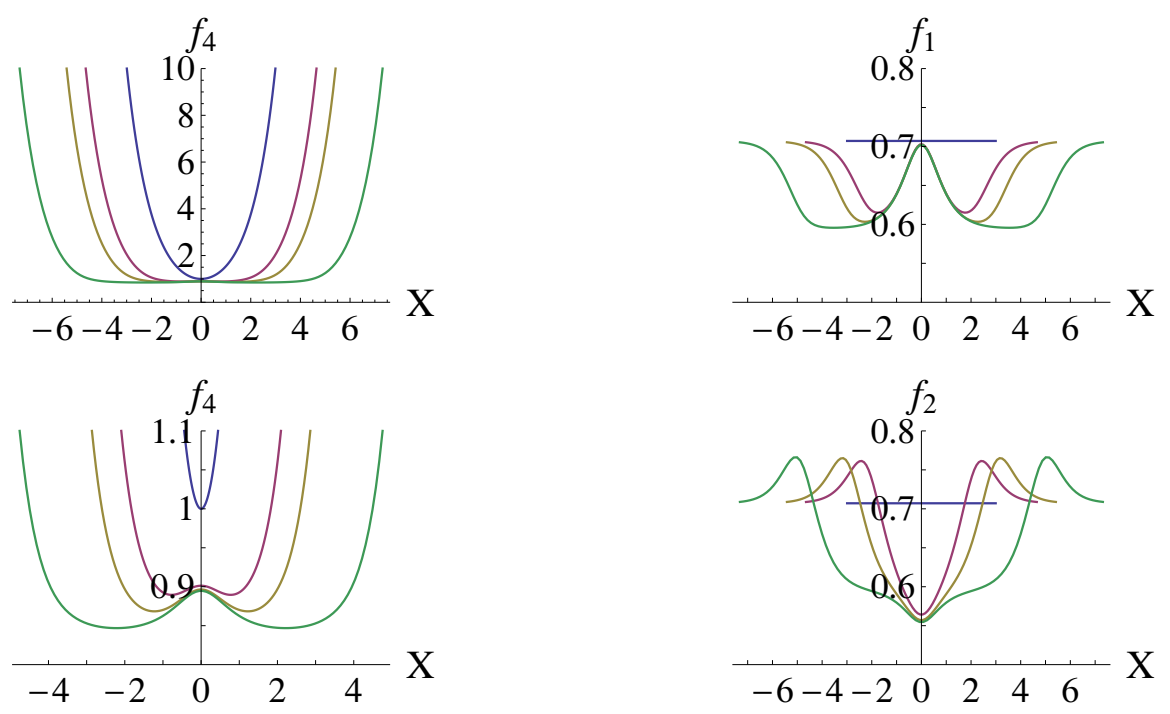

Figure 3. The warp factor along the $y=\pi / 4$ line for an NS5-brane stack (see section 3.3), with charge $Q_{N S 5}=16 \pi^{2} \gamma$ where $\gamma=0,1 / 2,1,5$ (blue, purple, yellow and green curves, respectively). The horizontal axis gives the invariant distance from the strip center. The asymptotic $\mathrm{AdS}_{5}$ radius has been set to one, and the asymptotic dilaton has been set to zero. Note, as can be seen in the lower left figure, that the local maximum of $f_{4}$ does not exceed the asymptotic value of the $\mathrm{AdS}_{5}$ radius.
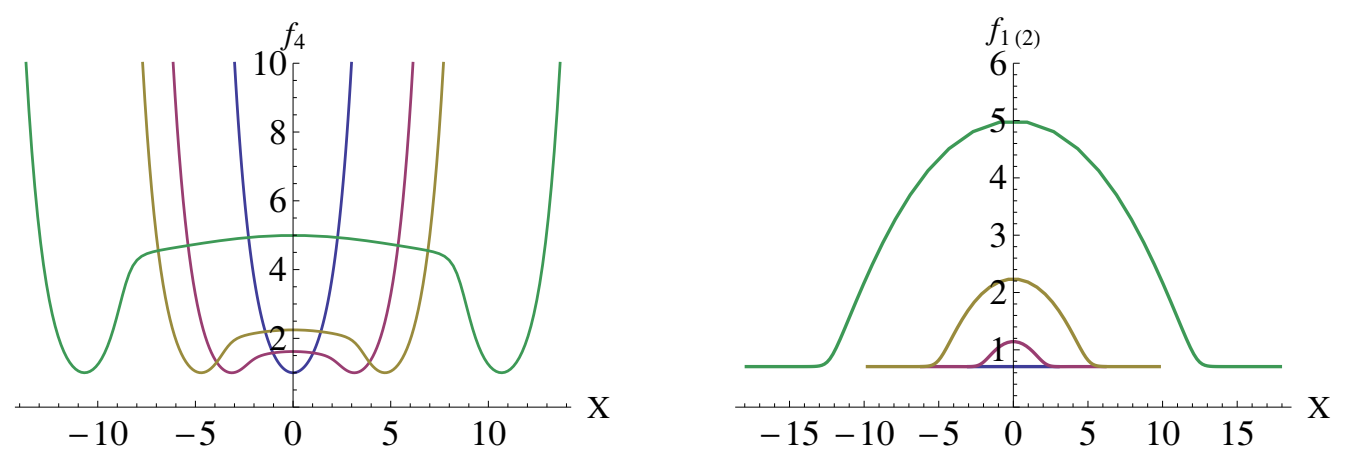

Figure 4. Warp factor along the $y=\pi / 4$ line for intersecting D5-brane and NS5-brane stacks (see section 3.3), with brane charges $Q_{D 5}=Q_{N S 5}=16 \pi^{2} \gamma$ where $\gamma=0,1 / 2,1,5$ (blue, purple, yellow and green curves, respectively). The conventions are the same as in figure 3 . Note that on the line $y=\pi / 4$ the $S^{2}$ warp factors are identical $\left(f_{1}=f_{2}\right)$.

on the 2-spheres, the operator $\left(m^{2}+2\right)$ is the Laplace-Beltrami operator on the strip $\Sigma$, with metric $\left(2 \rho / f_{4}\right)^{2} d z d \bar{z}$ and with Neumann boundary conditions. As a check, we will diagonalize this operator to recover the well-known $\operatorname{AdS}_{5} \times \mathrm{S}^{5}$ mass spectrum. In the next section we will analyze the first non-trivial example, that of the supersymmetric Janus solution.

\subsection{Reduction of the eigenmode equation}

The eigenvalue equation (2.20) for spin-2 modes depends only, as we have seen, on the warp factor and the internal-space metric. For the interface solutions of D'Hoker et al these can 
be read from the general expression (3.4),

$$
A=\ln f_{4}, \quad \hat{g}_{a b} d y^{a} d y^{b}=f_{1}^{2} d s_{\mathrm{S}_{1}^{2}}^{2}+f_{2}^{2} d s_{\mathrm{S}_{2}^{2}}^{2}+4 \rho^{2} d z d \bar{z} .
$$

Exploiting the background isometries, we may, furthermore, expand the wavefunction $\psi$ in a basis of spherical harmonics, i.e. in self-explanatory notation

$$
\psi\left(y^{a}\right)=Y_{l_{1} m_{1}} Y_{l_{2} m_{2}} \psi_{l_{1} l_{2}}(z, \bar{z}),
$$

where we have suppressed the dependence of $Y_{l m}$ on the sphere coordinates. Inserting these expressions in (2.20) leads to the following eigenvalue equation:

$$
-f_{4}^{2}\left[\frac{\delta^{i j}}{4 \rho^{2}} \partial_{i} \partial_{j}+\frac{\delta^{i j}}{2 \rho^{2}}\left(\partial_{i} \ln \left(f_{4}^{2} f_{1} f_{2}\right)\right) \partial_{j}-\frac{l_{1}\left(l_{1}+1\right)}{f_{1}^{2}}-\frac{l_{2}\left(l_{2}+1\right)}{f_{2}^{2}}\right] \psi_{l_{1} l_{2}}=m^{2} \psi_{l_{1} l_{2}}
$$

with $z \equiv y^{1}+i y^{2}$ the complex coordinate of the strip, and $i, j=1,2$. The lowest mass eigenvalue corresponds to a wavefunction with $l_{1}=l_{2}=0$. Much of our analysis will be restricted to this class of wavefunctions.

The $l_{1}=l_{2}=0$ equation takes a simple form if expressed in terms of the harmonic functions $h_{1}$ and $h_{2}$. This follows from the identities (3.11), which imply in particular

$$
f_{4}^{2} f_{1} f_{2}=4\left|h_{1} h_{2}\right|, \quad \text { and } \quad \frac{\rho^{2}}{f_{4}^{2}}=\left|\frac{W}{2 h_{1} h_{2}}\right|=-\frac{\partial \bar{\partial}\left(h_{1} h_{2}\right)}{2 h_{1} h_{2}} .
$$

In the second equation we have used the fact that $W / h_{1} h_{2}$ is a negative-definite real function in the interior of $\Sigma$. From the above identities one gets

$$
\begin{aligned}
0 & =\partial \bar{\partial} \psi_{00}+\left(\partial \ln \left(f_{4}^{2} f_{1} f_{2}\right)\right) \bar{\partial} \psi_{00}+\left(\bar{\partial} \ln \left(f_{4}^{2} f_{1} f_{2}\right)\right) \partial \psi_{00}+m^{2} \frac{\rho^{2}}{f_{4}^{2}} \psi_{00} \\
& =\partial \bar{\partial} \psi_{00}+\left(\partial \ln \left(h_{1} h_{2}\right)\right) \bar{\partial} \psi_{00}+\left(\bar{\partial} \ln \left(h_{1} h_{2}\right)\right) \partial \psi_{00}-m^{2} \frac{W}{2 h_{1} h_{2}} \psi_{00}
\end{aligned}
$$

Multiplying now by $\left(h_{1} h_{2}\right)$ and rearranging various terms puts the eigenvalue equation in the following elegant form:

$$
\frac{2 h_{1} h_{2}}{W} \partial \bar{\partial} \tilde{\psi}_{00}=\left(m^{2}+2\right) \tilde{\psi}_{00}, \quad \text { where } \quad \tilde{\psi}_{00} \equiv h_{1} h_{2} \psi_{00} .
$$

The operator $\left(m^{2}+2\right)$ for this class of $s$-wave excitations is thus a Laplace-Beltrami operator on the strip, with the effective metric $\left(2 \rho / f_{4}\right)^{2} d z d \bar{z}$. Note, as a check, that a constant wavefunction $\psi_{00}$ implies $\tilde{\psi}_{00}=h_{1} h_{2}$, which solves the above equation for zero mass in accordance with the general expectations. This would-be massless graviton is not, however, a normalizable mode, as will become clear in a minute.

The reduced equation (5.6) is manifestly invariant under the $S$-duality transformation, which exchanges $h_{1}$ with $h_{2}$. This should be so, because $S$-duality does not affect the (Einstein-frame) metric. Independent rescalings of $h_{1}$ and $h_{2}$, which change the values of the radius and the dilaton in the asymptotic $\mathrm{AdS}_{5} \times \mathrm{S}^{5}$ regions, are also symmetries of the above eigenvalue problem. This is a consequence of the scale covariance of the twoderivative supergravity action, and of the special form of the universal dilaton coupling. 
For completeness, let us also write down the equation for arbitrary values of the angular momenta, $l_{1}$ and $l_{2}$, on the two spheres:

$$
\left[\frac{2 h_{1} h_{2}}{W} \partial \bar{\partial}-\frac{N_{1}}{W h_{1}^{3} h_{2}} l_{1}\left(l_{1}+1\right)-\frac{N_{2}}{W h_{2}^{3} h_{1}} l_{2}\left(l_{2}+1\right)\right] \tilde{\psi}_{l_{1} l_{2}}=\left(m^{2}+2\right) \tilde{\psi}_{l_{1} l_{2}},
$$

where $\tilde{\psi}_{l_{1} l_{2}}=h_{1} h_{2} \psi_{l_{1} l_{2}}$ as above.This is again $S$-duality invariant, if one exchanges $l_{1}$ with $l_{2}$. Note that $W h_{1} h_{2}$ is negative-definite within the strip, so as expected non-vanishing angular momenta add a positive term to the mass-squared operator.

To fully specify the spectral problem, we must also discuss the space of allowed functions on the strip. Recall that generic points on the strip boundary are regular interior points of the 10-dimensional geometry, and $\operatorname{Im} z=\epsilon \rightarrow 0$ is the radial polar coordinate of a local 3-dimensional coordinate patch. Requesting that the metric perturbation be analytic in the local Cartesian coordinates therefore implies

$$
\psi_{00}=\text { constant }+O\left(\epsilon^{2}\right), \quad \text { and } \quad \psi_{l_{1} l_{2}}=O(\epsilon) \text { if } l_{1}+l_{2} \neq 0
$$

Thus $\psi_{00}$ has a Neumann boundary condition on the real axis, while for all other $\psi_{l_{1} l_{2}}$ the condition is Dirichlet. The same conditions apply to the upper boundary of the strip. As for the behavior near singular points and at infinity, this is fixed by requiring normalizability of the wavefunction. From the general expression (2.25) for the norm one finds

$$
\|\psi\|^{2}=\int_{\Sigma} d^{2} z\left(4 \rho^{2} f_{1}^{2} f_{2}^{2} f_{4}^{2}\right)\left|\psi_{l_{1} l_{2}}\right|^{2}
$$

where the integration runs over the strip $\Sigma$. Using the identities (5.4) one arrives then at the following reduced expression:

$$
\|\psi\|^{2}=\int_{\Sigma} d^{2} z\left|W h_{1} h_{2}\right|\left|\psi_{l_{1} l_{2}}\right|^{2}=\int_{\Sigma} d^{2} z\left|\frac{W}{h_{1} h_{2}}\right|\left|\tilde{\psi}_{l_{1} l_{2}}\right|^{2}
$$

This norm guarantees that the Laplace-Beltrami operator is self-adjoint. The integral must, in particular, converge in the asymptotic $\mathrm{AdS}_{5} \times \mathrm{S}^{5}$ regions, as well as in the vicinity of five-branes. The boundary conditions (5.8) together with the above norm determine the space of allowed spin-2 excitations in the background of all interface solutions.

We mention for future reference the equivalent Schrödinger problem (2.21)-(2.22), which is defined on the full six-dimensional internal space with metric

$$
\begin{aligned}
\bar{g}_{a b} d y^{a} d y^{b} & =f_{4}^{-2}\left(f_{1}^{2} d s_{\mathrm{S}_{1}^{2}}^{2}+f_{2}^{2} d s_{\mathrm{S}_{2}^{2}}^{2}+4 \rho^{2} d z d \bar{z}\right) \\
& =\left|\frac{h_{1}^{2} W}{N_{1}}\right| d s_{\mathrm{S}_{1}^{2}}^{2}+\left|\frac{h_{2}^{2} W}{N_{2}}\right| d s_{\mathrm{S}_{2}^{2}}^{2}+\left|\frac{2 W}{h_{1} h_{2}}\right| d z d \bar{z} .
\end{aligned}
$$

Though the analog potential $V(y)=f_{4}^{-4} \bar{\square}_{y} f_{4}^{4}$ can help in some cases one's intuition, we will not use it in the present work. The reduced form of the equation will be more convenient for our purposes here. 


\subsection{The case of $\mathrm{AdS}_{5} \times \mathrm{S}^{5}$}

To verify our formulae, and as a simple warm-up exercise, let us first analyze the pure $\mathrm{AdS}_{5} \times \mathrm{S}^{5}$ geometry. Writing $z \equiv x+i y$, one has:

$$
\frac{W}{2 h_{1} h_{2}}=-\frac{1}{4 \cosh ^{2} x}, \quad \text { and } \quad h_{1} h_{2}=2 \alpha_{1} \alpha_{2} \cosh ^{2} x \sin 2 y .
$$

We focus on the wavefunctions $\psi_{00}$ which, as we have just seen, must obey Neumann boundary conditions on the infinite strip. Their $y$-dependence can thus be decomposed in the basis of functions $\{\cos (2 n y)\}$ for $n=0,1,2 \cdots$. From the form (5.6) of the wave operator, one guesses the following form of orthogonal eigenfunctions:

$$
\psi_{00}(x, y)=e^{2 i n y}\left(\sum_{k=0}^{n} e^{-4 i k y}\right) \chi_{n}(x) \Longrightarrow \tilde{\psi}_{00}(x, y)=\sin [2(n+1) y] \tilde{\chi}_{n}(x),
$$

where $\tilde{\chi}_{n}(x) \equiv 2 \alpha_{1} \alpha_{2} \cosh ^{2} x \chi_{n}(x)$. With this ansatz the eigenvalue equation reduces to an ordinary differential equation for the function $\tilde{\chi}_{n}(x)$,

$$
-\cosh ^{2} x\left[\frac{d^{2}}{d x^{2}}-4(n+1)^{2}\right] \tilde{\chi}_{n}=\left(2+m^{2}\right) \tilde{\chi}_{n} .
$$

This is the Legendre equation, as can be recognized after the change of variables $\tanh x \equiv t$ which brings it to the canonical form [65]

$$
\left[\frac{d}{d t}\left(1-t^{2}\right) \frac{d}{d t}-\frac{4(n+1)^{2}}{1-t^{2}}+\kappa(\kappa+1)\right] \tilde{\chi}_{n}=0, \quad \text { where } \kappa(\kappa+1) \equiv 2+m^{2} .
$$

The general solution of the Legendre equation is a linear combination

$$
\tilde{\chi}_{n, \kappa}(t)=c_{1} P_{\kappa}^{2 n+2}(t)+c_{2} Q_{\kappa}^{2 n+2}(t)
$$

where $c_{1}, c_{2}$ are arbitrary coefficients, and $P_{\kappa}^{2 n+2}$ and $Q_{\kappa}^{2 n+2}$ are the associated Legendre functions of, respectively, the first and the second kind. To find the normalizable eigenmodes, note that in terms of the variable $-1<t<1$ the criterion (5.10) reads

$$
\int_{-1}^{1} d t\left|\tilde{\chi}_{n, \kappa}\right|^{2}<\infty
$$

At $t \rightarrow 1$ the Legendre function $Q_{\kappa}^{2 n+2}$ has a pole of order $n+1$, while $P_{\kappa}^{2 n+2}$ has a zero of the same order [65]. Convergence of the integral in this limit requires therefore that $c_{2}=0$. At $t \rightarrow-1$, on the other hand, the function $P_{\kappa}^{2 n+2}$ has a pole of order $n+1$, but with residue proportional to $\sin (\kappa \pi)$. To avoid the pole we must thus choose $\kappa \in \mathbb{Z}$. Since $\kappa$ and $-\kappa-1$ correspond to the same value of the mass, we may actually restrict $\kappa$ to the natural integers. Finally, Rodrigues' formula

$$
\tilde{\chi}_{n, \kappa} \propto P_{\kappa}^{2 n+2}(t)=\frac{1}{2^{\kappa} \kappa !}\left(1-t^{2}\right)^{n+1} \frac{d^{\kappa+2 n+2}}{d t^{\kappa+2 n+2}}\left(t^{2}-1\right)^{\kappa},
$$


shows that when $\kappa<2 n+2$ our would-be solution is identically zero. This leads then to the following spectrum of normalizable modes:

$$
m^{2}=(\kappa-1)(\kappa+2), \quad \text { for } \kappa \text { integer } \geq 2 n+2, \text { and } n=0,1,2 \cdots .
$$

The lowest-lying spin-2 excitation is as expected massive, with $m^{2}=4$.

The above spectrum corresponds to eigenmodes which are constant on both 2-spheres of the background metric (3.4). In the case at hand these 2-spheres make up, together with the $y$-interval, the 5 -sphere of the $\operatorname{AdS}_{5} \times \mathrm{S}^{5}$ solution. Thus all modes can be organized in representations of the bigger symmetry group $\mathrm{SO}(6)$. As can be easily checked, the states $(n, \kappa)$ belong to the symmetric tensor product of $n$ vector representations of $\mathrm{SO}(6)$. Their partners in this same representation, for $n \neq 0$, have a non-trivial dependence on the coordinates of the 2 -spheres, which is given by the 5 -sphere harmonics.

The $n=0$ states are $\mathrm{SO}(6)$ singlets, and there is one such state for each $\kappa=2,3 \cdots$. This decomposition of the $\mathrm{AdS}_{5}$ graviton into $\mathrm{AdS}_{4}$ eigenstates agrees with the fivedimensional analysis of Karch and Randall [17]. States with the same $n$ and different $\kappa$ are related by conformal, $\mathrm{SO}(2,4)$ transformations. Note, however, also that for given $\kappa$ there are $[\kappa / 2]$ different $\mathrm{SO}(6)$ representations with equal mass. This degeneracy is not accidental - it is due to the fact that all graviton states belong to a single unitary irreducible representation of the supergroup $\operatorname{PSU}(2,2 \mid 4)$.

\section{Spectrum of supersymmetric Janus}

We will now study the spectrum of spin-2 excitations in the supersymmetric Janus background. The key observation is that the $l_{1}=l_{2}=0$ eigenmode equation is in this case separable, and it reduces to an ordinary differential equation with four regular singular points. We will solve this equation numerically, and exhibit the spectrum as a function of the dilaton-jump parameter $\Delta \phi$. The result confirms the expectations from section 4 , in particular in the limit of large $\Delta \phi$ in which the interface spreads out to a large nearly-flat extra dimension with a linearly-varying dilaton.

\subsection{Heun's equation}

Although the Janus geometry does not factorize, it follows from eqs. (4.13) that the ratio $\left(W / h_{1} h_{2}\right)$ is $y$-independent. Thus, at least for the $l_{1}=l_{2}=0$ modes, we can use the ansatz (5.13) to separate the variables $x$ and $y$. The eigenvalue equation (5.6) reduces then to the following ordinary differential equation:

$$
-\frac{\cosh \Delta \phi+\cosh 2 x}{2 \cosh \Delta \phi}\left[\frac{d^{2}}{d x^{2}}-4(n+1)^{2}\right] \tilde{\chi}_{n}=\left(2+m^{2}\right) \tilde{\chi}_{n},
$$

where here $\tilde{\chi}_{n} \equiv \alpha_{1} \alpha_{2}(\cosh \Delta \phi+\cosh 2 x) \chi_{n}$. Changing again to the variable $t \equiv \tanh x$, and defining the convenient "Janus parameter"

$$
\left(\operatorname{coth} \frac{\Delta \phi}{2}\right)^{2} \equiv \xi \in(1, \infty]
$$


brings the above equation to the equivalent form

$$
\left[\frac{d}{d t}\left(1-t^{2}\right) \frac{d}{d t}-\frac{4(n+1)^{2}}{1-t^{2}}+\frac{\left(2+m^{2}\right)(1+\xi)}{\xi-t^{2}}\right] \tilde{\chi}_{n}=0 .
$$

This is an equation with five regular singular points, at $t= \pm 1, t= \pm \sqrt{\xi}$ and $t=\infty$, all of which lie outside the open interval $(-1,1)$ where $\tilde{\chi}_{n}(t)$ should be defined. The $\operatorname{AdS}_{5} \times S^{5}$ equation is recovered in the limit $\Delta \phi \rightarrow 0$, i.e. $\xi \rightarrow \infty$.

The number of singularities of (6.3) can be reduced by exploiting its symmetry under the reflection $t \rightarrow-t$. Thanks to this symmetry, the eigenfunctions can be chosen to be either even or odd functions of $t$. Changing variable to $s=t^{2}$ leads to

$$
\left[4 s(1-s) \frac{d^{2}}{d s^{2}}+2(1-3 s) \frac{d}{d s}-\frac{4(n+1)^{2}}{1-s}+\frac{\left(2+m^{2}\right)(1+\xi)}{\xi-s}\right] \tilde{\chi}_{n}=0,
$$

with even/odd solutions distinguished by their behavior at $s \rightarrow 0$ :

$$
\tilde{\chi}_{n}^{\text {even }}=\text { analytic }, \quad \tilde{\chi}_{n}^{\text {odd }}=(\text { analytic }) \times \sqrt{s} .
$$

Equation (6.4) has four regular singular points, at $s=0,1, \xi$ and $\infty$. [That the point $s=\infty$ is regular can be seen after a change of variables $s^{\prime}=1 / s$.] Such differential equations are known in the mathematics literature as Heun's equations $[40,66]$. They arise in a variety of physical contexts, for instance as eigenvalue equations in the background of the KerrNewman-de Sitter black hole [67, 68], of large AdS black holes in five dimensions [69], of the toric Sasaki-Einstein manifolds $L^{a, b, c}$ [70], or in the study of RG flows between 2D CFTs [71] and of slowly-rotating homogeneous stars [72] (for more physics applications see the references in [40,73]). Heun's equation is the next in the series of canonical Fuchsian equations on the Riemann sphere, after the hypergeometric equation which has three regular singular points.

Near a regular singular point, the two (local) solutions of a Fuchsian equation behave as $\left(s-s_{0}\right)^{\nu_{1}} f_{1}(s)$ and $\left(s-s_{0}\right)^{\nu_{2}} f_{2}(s)$, where $\nu_{1}$ and $\nu_{2}$ are called the singularity exponents, and $f_{1}, f_{2}$ are analytic at $s_{0} .{ }^{12}$ In the case at hand, the exponents at the singular point $s_{0}=0$ are $\nu_{1}=0$ and $\nu_{2}=1 / 2$, corresponding respectively to the even and the odd solutions. At the singular point $s_{0}=1$, on the other hand, the exponents are $\pm(n+1)$. Since the normalizability condition (5.10) requires that

$$
\int_{0}^{1} \frac{d s}{\sqrt{s}} \frac{\xi}{\xi-s}\left|\tilde{\chi}_{n}\right|^{2}<\infty
$$

solutions singular at $s_{0}=1$ must be excluded. Thus, to determine the spectrum we need to find the values of $m^{2}$ for which the even (odd) solutions of (6.4) are analytic at $s=1$. [Solutions that are analytic in a region encompassing two singular points, are called Heun's functions in the mathematics literature.]

For the generic Heun equation it is not known how to solve this two-point connection problem in closed form. Semi-analytic expressions for $\tilde{\chi}_{n}$ can be written down as series of

\footnotetext{
${ }^{12}$ Except when $\left(\nu_{2}-\nu_{1}\right)$ is a non-negative integer, in which case $f_{1}$ has a logarithmic singularity.
} 
hypergeometric functions whose coefficients obey simple recurrence relations [40]. These series converge in a region around a given singular point, so to determine $m^{2}$ in the case at hand one must match the expansions around the points $s=0$ and $s=1$ (for an example of this procedure see $[67,68])$. For the purposes of this work, simpler numerical algorithms will be sufficient. Before turning to the numerical solution, we first bring equation (6.4) to the canonical Heun form

$$
\frac{d^{2} g}{d^{2} s}+\left(\frac{\gamma}{s}+\frac{\delta}{s-1}+\frac{\epsilon}{s-\xi}\right) \frac{d g}{d s}+\frac{\alpha \beta s-q}{s(s-1)(s-\xi)} g=0,
$$

where the parameters must obey the constraint $\gamma+\delta+\epsilon=\alpha+\beta+1$. Our equation is brought to this form by the wavefunction redefinition

$$
\tilde{\chi}_{n}(s) \equiv(1-s)^{n+1}(\xi-s) g(s),
$$

and with the following identification of the parameters:

$$
\begin{aligned}
\alpha=n+2, \quad \beta & =n+\frac{5}{2}, \quad \gamma=\frac{1}{2}, \quad \delta=2 n+3, \quad \epsilon=2, \\
4 q & =\xi(2 n+1)(2 n+4)-(1+\xi) m^{2} .
\end{aligned}
$$

Note that the roles of $\alpha$ and $\beta$ in the canonical form of Heun's equation can be swapped. Note also that since the exponents of $\tilde{\chi}_{n}$ at $s=1$ were $\pm(n+1)$, those of $g$ at this singular point are $-2 n-2$ and 0 . The normalizable solutions have $g(s)$ analytic at $s=1$, and analytic or with a square-root singularity at $s=0$.

\subsection{Numerical solution}

The Heun equation admits power series solutions around each of its singular points. We write the power-series solution around the point $s=0$ as

$$
g_{1}\left(n, m^{2}, \xi ; s\right) \equiv H l(\alpha, \beta, \gamma, \delta ; q ; \xi ; s)=\sum_{i=0}^{\infty} c_{i} s^{i} \quad \text { with } \quad c_{0}=1
$$

The $c_{i}$ are then determined by the three-term recursion relation

$$
\begin{aligned}
& 0=-q c_{0}+\xi \gamma c_{1}, \\
& 0=P_{i} c_{i-1}-\left(Q_{i}+q\right) c_{i}+R_{i} c_{i+1},
\end{aligned}
$$

where the coefficients in the lower line are given by

$$
\begin{aligned}
P_{i} & =(i-1+\alpha)(i-1+\beta), \\
Q_{i} & =i[(i-1+\gamma)(1+\xi)+\xi \delta+\epsilon], \\
R_{i} & =(i+1)(i+\gamma) \xi
\end{aligned}
$$

for $i=1,2 \cdots$. The second, linearly-independent solution of Heun's equation (6.7) can then be written in terms of the same function $\mathrm{Hl}$,

$$
g_{2}\left(n, m^{2}, \xi ; s\right)=s^{1-\gamma} H l\left(\alpha^{\prime}, \beta^{\prime}, 2-\gamma, \delta ; q^{\prime} ; \xi ; s\right)
$$


with the following transformed arguments:

$$
\alpha^{\prime}=\alpha-\gamma+1, \quad \beta^{\prime}=\beta-\gamma+1, \quad q^{\prime}=q+(1-\gamma)(\epsilon+\xi \delta) .
$$

Recall that $n$ was a label for the Fourier modes in the $y$ direction, $\xi$ parametrizes the dilaton jump, the $A d S_{4}$ mass is $m^{2}$, and the remaining parameters are given in eq. (6.9). Note also that for $\gamma=1 / 2$ the two solutions correspond to the two possible behaviors (6.5), i.e. $g_{1}$ is the even and $g_{2}$ the odd solution. Our problem is to find the values of $m^{2}$ for which these solutions are regular at $s=1$.

The asymptotic behavior near $s=1$ can be studied most easily by making a linear fractional change of variable

$$
\hat{s}=\frac{\xi(s-1)}{s-\xi},
$$

and a redefinition of the function $\hat{g}(\hat{s})=(\hat{s}-\xi)^{-\alpha} g(\hat{s})$. This Möbius transformation maps Heun's equation into itself, swapping the singular points $0 \leftrightarrow 1$ and $\xi \leftrightarrow \infty$, and transforming the parameters as follows:

$$
\begin{array}{lll}
\hat{\alpha}=\alpha, & \hat{\beta}=1+\alpha-\epsilon, & \hat{\delta}=\gamma,
\end{array} \quad \hat{\gamma}=\delta,
$$

The solution of the original equation that is regular at $s=1$ can be written as

$$
g_{\mathrm{reg}}\left(n, m^{2}, \xi ; s\right)=(\xi-\hat{s})^{\hat{\alpha}} H l(\hat{\alpha}, \hat{\beta}, \hat{\delta}, \hat{\gamma}, \hat{\epsilon} ; \hat{q} ; \xi ; \hat{s}),
$$

while the second solution is singular and must be excluded. The normalizable wavefunctions are thus the ones for which $g_{1}=A_{1} g_{\text {reg }}$ or $g_{2}=A_{2} g_{\text {reg }}$, where $A_{1}$ and $A_{2}$ are proportionality constants. These conditions determine the allowed values of $m^{2}$ for given values of $n$ and $\xi$.

There are no general techniques which are currently known for the analytic solution of this problem. ${ }^{13}$ The following simple numerical algorithm will be, however, sufficient for our purposes here. Using the recursion relation (6.11) we compute the series expansions of $g_{1,2}$ and $g_{\mathrm{reg}}$, around the singular points 0 and 1, to a high order. We then impose the following matching condition for the even or odd solutions:

$$
\left.\frac{\partial}{\partial s} \ln \frac{g_{1}}{g_{\text {reg }}}\right|_{s_{0}}=0 \quad \text { or }\left.\quad \frac{\partial}{\partial s} \ln \frac{g_{2}}{g_{\text {reg }}}\right|_{s_{0}}=0
$$

where $s_{0}$ is a midway point at which all series expansions are convergent. The results for $n=0$ and different values of $\xi$ are shown in figure 5 . As can be seen, all the masses decrease monotonically as $\xi$ increases, and they all approach zero as $\xi$ approaches 1 . The behavior for $\xi$ close to 1 , corresponding to large $\Delta \phi$, is shown in figure 6 . As discussed in section 4.2 , a nearly-flat fifth dimension grows to size $\sim \Delta \phi$ in this limit. Thus the spacing between the masses of neighboring modes should be constant and of order $\Delta \phi^{-1}$, and this is confirmed by the numerical analysis.

\footnotetext{
${ }^{13} \mathrm{An}$ approach along the lines of reference [67, 68] may, however, be worth trying.
} 


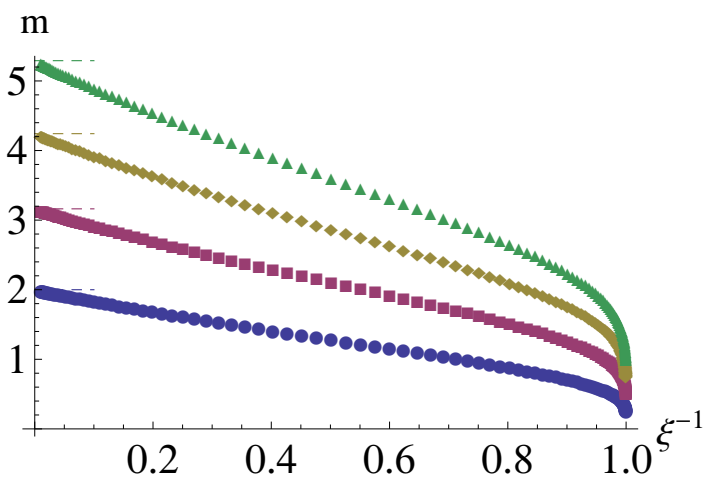

Figure 5. Numerical results for the $\mathrm{AdS}_{4}$ masses of the first four normalizable modes for $n=0$. The dashed lines at $\xi \simeq \infty$ correspond to the $A d S_{5}$ values $m=2, \sqrt{10}, \sqrt{18}, \sqrt{28}$.

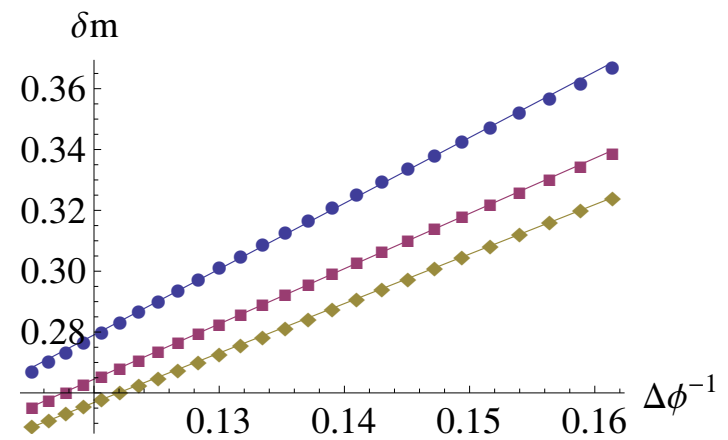

Figure 6. Numerical results for the mass spacing, $\delta m$, as a function of $\Delta \phi^{-1}$ for large $\Delta \phi$. Shown are the spacings between the first two modes (blue circles), between the third and the second mode (purple squares) and between the fourth and third modes (yellow diamonds). The solid lines are a linear fit to the numerical results.

This limiting situation can be analyzed more easily by going back to the original form (6.1) of the eigenmode equation. Expanding this equation for large $\Delta \phi$ gives

$$
\left(-\frac{1}{2} \frac{d^{2}}{d x^{2}}+2 e^{2|x|-\Delta \phi}\right) \tilde{\chi}_{0} \simeq m^{2} \tilde{\chi}_{0}
$$

where we have set $n=0$ and we assumed that $m \ll 1$. The solutions are plane waves, $\tilde{\chi}_{0} \simeq e^{ \pm \sqrt{2 m} x}$, cutoff by the exponentially-rising potential at $x \simeq \pm \Delta \phi / 2$. The potential wall discretizes the low-lying mass spectrum in units of $\Delta \phi^{-1}$ as advertized. This completes our analysis of the Janus spectrum.

\section{Discussion}

A lot of effort has gone, in recent years, into finding a string-theory realization of the socalled model-I of Randall and Sundrum [74]. A popular embedding [75], for example, uses 
the Klebanov-Strassler throat geometry [76] capped-off by a compact Calabi-Yau manifold. Despite some notable differences, the gravitational physics in this setting resembles that of ordinary Kaluza-Klein compactifications (see for instance refs. [77-79]). There exists, in particular, a normalizable zero mode and a finite mass gap for the higher spin-2 excitations.

The backgrounds considered in this work are qualitatively different, since the internal space is not compact and the graviton has a non-vanishing $\mathrm{AdS}_{4}$ mass. They resemble in this respect the thin-brane geometry of the Karch-Randall model [17], a prototypical example of (quasi-)localized gravity. A crucial aspect of this model is that the excited modes contribute to the long-range force, but this contribution is suppressed by an exponentiallyrising warp factor. The lack of a compelling effective theory for massive gravity in four dimensions probably means that efforts to embed this model in string theory are doomed to fail. Nevertheless, given the importance of the question, even failure can be instructive and all avenues should be pursued. The exact interface backgrounds of D'Hoker et al [19, 20] allow us to revisit this question in a controlled setting, and this has motivated the present work.

One of our tasks in this paper was to carefully set up the spectral problem in the above background geometries, in sections 2 and 5 . The exercise is straightforward, but performing carefully all steps allowed us to tie up loose ends, and to emphasize the differences with the more familiar cases of flat-brane warped geometries and of direct-product $\mathrm{AdS}_{4}$ compactifications. In this paper we have solved this spectral problem only in the simplest case of the supersymmetric Janus solution, for which the eigenmode equation is separable. Our discussion is, however, also the starting point for the study of the more involved five-brane solutions, whose spectral problem is inherently 2-dimensional and thus requires more sophisticated numerical and analytic techniques [41]. Finally, although much of our discussion has been guided by the issue of gravity localization, the spectral problem for spin-2 modes is of broader interest from the perspective of defect CFTs. One question, to which we hope to return, is the precise relation between this spectrum and the energy-transfer properties of the holographically-dual defect walls.

\section{Acknowledgments}

We have benefited from discussions with Benjamin Assel, Iosif Bena, Cedric Deffayet, Eric D'Hoker, Elias Kiritsis, Joao Penedones, Tassos Petkou, Giuseppe Policastro, Slava Rychkov, Paul Townsend, Jan Troost and Ed Witten. J.E. is supported by the FWO Vlaanderen, Project No. G.0235.05, and by the "Federal Office for Scientific, Technical and Cultural Affairs through the Inter-University Attraction Poles Programme," Belgian Science Policy P6/11-P.

\section{A Summary of type IIB supergravity}

We briefly review here the type-IIB supergravity Bianchi identities and field equations, as well as the supersymmetry variations, for vanishing fermionic fields. Our conventions are those used in $[19,20]$, except for a rescaling of the 4 -form potential by a factor 4 so 
as to agree with the standard conventions. The bosonic fields are the metric $g_{M N}$, the complex axion-dilaton scalar $B$, the complex 2 -form $B_{(2)}$ and the real 4 -form $C_{(4)}$. To simplify the form of the field equations and Bianchi identities we introduce the following composite fields:

$$
P=f^{2} d B, \quad Q=f^{2} \operatorname{Im}(B d \bar{B}) \quad \text { where } f^{2}=\left(1-|B|^{2}\right)^{-1},
$$

and the complex 3 -form and real 5-form field strengths

$$
\begin{aligned}
G & =f\left(F_{(3)}-B \bar{F}_{(3)}\right), \\
F_{(5)} & =d C_{(4)}+\frac{i}{4}\left(B_{(2)} \wedge \bar{F}_{(3)}-\bar{B}_{(2)} \wedge F_{(3)}\right),
\end{aligned}
$$

where $F_{(3)}=d B_{(2)}$. The scalar field $B$ is related to the complex string coupling $\tau$, the axion $\chi$, and the dilaton $\phi$ by

$$
B=\frac{1+i \tau}{1-i \tau}, \quad \tau=\chi+i e^{-2 \phi} .
$$

In terms of the composite fields $P, Q$, and $G$, the Bianchi identities read

$$
\begin{aligned}
& 0=d P-2 i Q \wedge P, \\
& 0=d Q+i P \wedge \bar{P}, \\
& 0=d G-i Q \wedge G+P \wedge \bar{G}, \\
& 0=d F_{(5)}-\frac{i}{2} G \wedge \bar{G} .
\end{aligned}
$$

The field strength $F_{(5)}$ is furthermore required to be self-dual,

$$
F_{(5)}=* F_{(5)} .
$$

The field equations are given by

$$
\begin{aligned}
0= & \nabla^{\mu} P_{\mu}-2 i Q^{\mu} P_{\mu}+\frac{1}{24} G_{\mu \nu \rho} G^{\mu \nu \rho} \\
0= & \nabla^{\rho} G_{\mu \nu \rho}-i Q^{\rho} G_{\mu \nu \rho}-P^{\rho} \bar{G}_{\mu \nu \rho}+\frac{2}{12} i F_{(5) \mu \nu \rho \sigma \kappa} G^{\rho \sigma \kappa}, \\
0= & R_{\mu \nu}-P_{\mu} \bar{P}_{\nu}-\bar{P}_{\mu} P_{\nu}-\frac{1}{96} F_{(5) \mu \rho \sigma \kappa \gamma} F_{(5) \nu}{ }^{\rho \sigma \kappa \gamma} \\
& -\frac{1}{8}\left(G_{\mu}{ }^{\rho \sigma} \bar{G}_{\nu \rho \sigma}+\bar{G}_{\mu}{ }^{\rho \sigma} G_{\nu \rho \sigma}\right)+\frac{1}{48} g_{\mu \nu} G^{\rho \sigma \kappa} \bar{G}_{\rho \sigma \kappa}
\end{aligned}
$$

Type-IIB supergravity is invariant under $\mathrm{SU}(1,1) \sim \mathrm{SL}(2, \mathbf{R})$ transformations which leave $g_{\mu \nu}$ and $C_{(4)}$ invariant, act as Möbius transformations on the field $B$, and transform linearly the 2-form $B_{(2)}$,

$$
\begin{aligned}
B & \rightarrow B^{s}=\frac{u B+v}{\bar{v} B+\bar{u}}, \\
B_{(2)} & \rightarrow B_{(2)}^{s}=u B_{(2)}+v \bar{B}_{(2)},
\end{aligned}
$$


with $u, v \in \mathbf{C}$ and $\bar{u} u-\bar{v} v=1$. In this non-linear realization of $\mathrm{SU}(1,1)$, the field $B$ takes values in the coset $\mathrm{SU}(1,1) / \mathrm{U}(1)_{q}$, and the fermions $\lambda$ and $\psi_{\mu}$ transform linearly under the isotropy gauge group $\mathrm{U}(1)_{q}$ with composite gauge field $Q$. The transformation rules for the composite fields are,

$$
\begin{aligned}
& P \rightarrow P^{s}=e^{2 i \theta} P, \\
& Q \rightarrow Q^{s}=Q+d \theta, \\
& G \rightarrow G^{s}=e^{i \theta} G,
\end{aligned}
$$

where the phase $\theta$ is defined by

$$
e^{i \theta}=\left(\frac{v \bar{B}+u}{\bar{v} B+\bar{u}}\right)^{\frac{1}{2}}
$$

Written like this the transformation rules clearly exhibit the $\mathrm{U}(1)_{q}$ gauge variation that accompanies the global $\mathrm{SU}(1,1)$ transformations.

Open Access. This article is distributed under the terms of the Creative Commons Attribution Noncommercial License which permits any noncommercial use, distribution, and reproduction in any medium, provided the original author(s) and source are credited.

\section{References}

[1] V.A. Rubakov and M.E. Shaposhnikov, Do we live inside a domain wall?, Phys. Lett. B 125 (1983) 136 [SPIRES].

[2] L. Randall and R. Sundrum, An alternative to compactification, Phys. Rev. Lett. 83 (1999) 4690 [hep-th/9906064] [SPIRES].

[3] G.R. Dvali, G. Gabadadze and M. Porrati, 4D gravity on a brane in $5 D$ Minkowski space, Phys. Lett. B 485 (2000) 208 [hep-th/0005016] [SPIRES].

[4] H. van Dam and M.J.G. Veltman, Massive and massless Yang-Mills and gravitational fields, Nucl. Phys. B 22 (1970) 397 [SPIRES].

[5] V.I. Zakharov, Linearized gravitation theory and the graviton mass, JETP Lett. 12 (1970) 312 [Pisma Zh. Eksp. Teor. Fiz. 12 (1970) 447].

[6] A.I. Vainshtein, To the problem of nonvanishing gravitation mass, Phys. Lett. B 39 (1972) 393 [SPIRES].

[7] D.G. Boulware and S. Deser, Inconsistency of finite range gravitation, Phys. Lett. B 40 (1972) 227 [SPIRES].

[8] D.G. Boulware and S. Deser, Can gravitation have a finite range?, Phys. Rev. D 6 (1972) 3368 [SPIRES].

[9] R. Gregory, V.A. Rubakov and S.M. Sibiryakov, Opening up extra dimensions at ultra-large scales, Phys. Rev. Lett. 84 (2000) 5928 [hep-th/0002072] [SPIRES].

[10] N. Arkani-Hamed, H. Georgi and M.D. Schwartz, Effective field theory for massive gravitons and gravity in theory space, Ann. Phys. 305 (2003) 96 [hep-th/0210184] [SPIRES]. 
[11] C. Deffayet and J.-W. Rombouts, Ghosts, strong coupling and accidental symmetries in massive gravity, Phys. Rev. D 72 (2005) 044003 [gr-qc/0505134] [SPIRES].

[12] V.A. Rubakov and P.G. Tinyakov, Infrared-modified gravities and massive gravitons, Phys. Usp. 51 (2008) 759 [arXiv:0802.4379] [SPIRES].

[13] E. Kiritsis and V. Niarchos, Interacting string multi-verses and holographic instabilities of massive gravity, Nucl. Phys. B 812 (2009) 488 [arXiv:0808.3410] [SPIRES].

[14] D. Blas, Aspects of infrared modifications of gravity, arXiv:0809.3744 [SPIRES].

[15] C. de Rham and G. Gabadadze, Selftuned massive spin-2, Phys. Lett. B 693 (2010) 334 [arXiv: 1006.4367] [SPIRES].

[16] L. Alberte, A.H. Chamseddine and V. Mukhanov, Massive gravity: exorcising the ghost, JHEP 04 (2011) 004 [arXiv: 1011.0183] [SPIRES].

[17] A. Karch and L. Randall, Locally localized gravity, JHEP 05 (2001) 008 [hep-th/0011156] [SPIRES].

[18] A. Karch and L. Randall, Localized gravity in string theory, Phys. Rev. Lett. 87 (2001) 061601 [hep-th/0105108] [SPIRES].

[19] E. D'Hoker, J. Estes and M. Gutperle, Exact half-BPS Type IIB interface solutions I: local solution and supersymmetric Janus, JHEP 06 (2007) 021 [arXiv: 0705.0022] [SPIRES].

[20] E. D'Hoker, J. Estes and M. Gutperle, Exact half-BPS type IIB interface solutions II: flux solutions and multi-Janus, JHEP 06 (2007) 022 [arXiv: 0705.0024] [SPIRES].

[21] J. Gomis and C. Romelsberger, Bubbling defect CFT's, JHEP 08 (2006) 050 [hep-th/0604155] [SPIRES].

[22] O. Lunin, Brane webs and 1/4-BPS geometries, JHEP 09 (2008) 028 [arXiv:0802.0735] [SPIRES].

[23] A. Karch and L. Randall, Open and closed string interpretation of SUSY CFT's on branes with boundaries, JHEP 06 (2001) 063 [hep-th/0105132] [SPIRES].

[24] O. DeWolfe, D.Z. Freedman and H. Ooguri, Holography and defect conformal field theories, Phys. Rev. D 66 (2002) 025009 [hep-th/0111135] [SPIRES].

[25] C. Bachas, J. de Boer, R. Dijkgraaf and H. Ooguri, Permeable conformal walls and holography, JHEP 06 (2002) 027 [hep-th/0111210] [SPIRES].

[26] C. Bachas and M. Petropoulos, Anti-de-Sitter D-branes, JHEP 02 (2001) 025 [hep-th/0012234] [SPIRES].

[27] J. Erdmenger, Z. Guralnik and I. Kirsch, Four-dimensional superconformal theories with interacting boundaries or defects, Phys. Rev. D 66 (2002) 025020 [hep-th/0203020] [SPIRES].

[28] D. Gaiotto and E. Witten, Janus configurations, Chern-Simons couplings, and the theta-angle in $N=4$ super Yang-Mills theory, JHEP 06 (2010) 097 [arXiv:0804.2907] [SPIRES].

[29] D. Gaiotto and E. Witten, S-duality of boundary conditions in $N=4$ super Yang-Mills theory, arXiv: 0807.3720 [SPIRES].

[30] E. Kiritsis, Product CFTs, gravitational cloning, massive gravitons and the space of gravitational duals, JHEP 11 (2006) 049 [hep-th/0608088] [SPIRES]. 
[31] O. Aharony, A.B. Clark and A. Karch, The CFT/AdS correspondence, massive gravitons and a connectivity index conjecture, Phys. Rev. D 74 (2006) 086006 [hep-th/0608089] [SPIRES].

[32] O. Aharony, O. DeWolfe, D.Z. Freedman and A. Karch, Defect conformal field theory and locally localized gravity, JHEP 07 (2003) 030 [hep-th/0303249] [SPIRES].

[33] M. Porrati, Mass and gauge invariance. IV: holography for the Karch-Randall model, Phys. Rev. D 65 (2002) 044015 [hep-th/0109017] [SPIRES].

[34] R. Bousso and L. Randall, Holographic domains of Anti-de Sitter space, JHEP 04 (2002) 057 [hep-th/0112080] [SPIRES].

[35] M.J. Duff and J.T. Liu, Complementarity of the Maldacena and Randall-Sundrum pictures, Class. Quant. Grav. 18 (2001) 3207 [Phys. Rev. Lett. 85 (2000) 2052] [hep-th/0003237] [SPIRES].

[36] M.J. Duff, J.T. Liu and H. Sati, Complementarity of the Maldacena and Karch-Randall pictures, Phys. Rev. D 69 (2004) 085012 [hep-th/0207003] [SPIRES].

[37] O. Aharony, D. Marolf and M. Rangamani, Conformal field theories in Anti-de Sitter space, JHEP 02 (2011) 041 [arXiv: 1011.6144] [SPIRES].

[38] C. Csáki, J. Erlich, T.J. Hollowood and Y. Shirman, Universal aspects of gravity localized on thick branes, Nucl. Phys. B 581 (2000) 309 [hep-th/0001033] [SPIRES].

[39] A. Brandhuber and K. Sfetsos, Non-standard compactifications with mass gaps and Newton's law, JHEP 10 (1999) 013 [hep-th/9908116] [SPIRES].

[40] A. Ronveaux, Heun's differential equations, Oxford University Press, Oxford U.K. (1995).

[41] B. Assel, C. Bachas and J. Estes, work in progress.

[42] M. Fierz, Force-free particles with any spin, Helv. Phys. Acta 12 (1939) 3 [SPIRES].

[43] M. Fierz and W. Pauli, On relativistic wave-equations for particles of arbitrary spin in an electromagnetic field, Proc. Roy. Soc. A 173 (1939) 221.

[44] I.L. Buchbinder, D.M. Gitman, V.A. Krykhtin and V.D. Pershin, Equations of motion for massive spin 2 field coupled to gravity, Nucl. Phys. B 584 (2000) 615 [hep-th/9910188] [SPIRES].

[45] S. Deser and A. Waldron, Stability of massive cosmological gravitons, Phys. Lett. B 508 (2001) 347 [hep-th/0103255] [SPIRES].

[46] S.M. Carroll, Spacetime and geometry: an introduction to general relativity, Addison-Wesley, San Fransisco U.S.A. (2004), p. 513 [SPIRES].

[47] M.P. Dabrowski, J. Garecki and D.B. Blaschke, Conformal transformations and conformal invariance in gravitation, Annalen Phys. 18 (2009) 13 [arXiv:0806.2683] [SPIRES].

[48] A. Polishchuk, Massive symmetric tensor field on AdS, JHEP 07 (1999) 007 [hep-th/9905048] [SPIRES].

[49] N. Kaloper, Bent domain walls as braneworlds, Phys. Rev. D 60 (1999) 123506 [hep-th/9905210] [SPIRES].

[50] E. Kiritsis and F. Nitti, On massless $4 D$ gravitons from $5 D$ asymptotically AdS space-times, Nucl. Phys. B 772 (2007) 67 [hep-th/0611344] [SPIRES]. 
[51] L. Girardello, M. Petrini, M. Porrati and A. Zaffaroni, Novel local CFT and exact results on perturbations of $N=4$ super Yang-Mills from AdS dynamics, JHEP 12 (1998) 022 [hep-th/9810126] [SPIRES].

[52] D.Z. Freedman, S.S. Gubser, K. Pilch and N.P. Warner, Renormalization group flows from holography supersymmetry and a c-theorem, Adv. Theor. Math. Phys. 3 (1999) 363 [hep-th/9904017] [SPIRES].

[53] A. Strominger, Open p-branes, Phys. Lett. B 383 (1996) 44 [hep-th/9512059] [SPIRES].

[54] D. Marolf, Chern-Simons terms and the three notions of charge, hep-th/0006117 [SPIRES].

[55] M. Cvetič, S. Griffies and H.H. Soleng, Local and global gravitational aspects of domain wall space-times, Phys. Rev. D 48 (1993) 2613 [gr-qc/9306005] [SPIRES].

[56] O. DeWolfe, D.Z. Freedman, S.S. Gubser and A. Karch, Modeling the fifth dimension with scalars and gravity, Phys. Rev. D 62 (2000) 046008 [hep-th/9909134] [SPIRES].

[57] E.G. Adelberger, B.R. Heckel and A.E. Nelson, Tests of the gravitational inverse-square law, Ann. Rev. Nucl. Part. Sci. 53 (2003) 77 [hep-ph/0307284] [SPIRES].

[58] C.D. Hoyle et al., Sub-millimeter tests of the gravitational inverse-square law, Phys. Rev. D 70 (2004) 042004 [hep-ph/0405262] [SPIRES].

[59] A. Miemiec, A power law for the lowest eigenvalue in localized massive gravity, Fortsch. Phys. 49 (2001) 747 [hep-th/0011160] [SPIRES].

[60] J. Garriga and T. Tanaka, Gravity in the brane-world, Phys. Rev. Lett. 84 (2000) 2778 [hep-th/9911055] [SPIRES].

[61] A. Kehagias and K. Sfetsos, Deviations from the $1 / r^{2}$ Newton law due to extra dimensions, Phys. Lett. B 472 (2000) 39 [hep-ph/9905417] [SPIRES].

[62] E. Kiritsis, N. Tetradis and T.N. Tomaras, Induced gravity on RS branes, JHEP 03 (2002) 019 [hep-th/0202037] [SPIRES].

[63] P. Callin and F. Ravndal, Higher order corrections to the Newtonian potential in the Randall-Sundrum model, Phys. Rev. D 70 (2004) 104009 [hep-ph/0403302] [SPIRES].

[64] D. Bazeia, A.R. Gomes and L. Losano, Gravity localization on thick branes: a numerical approach, Int. J. Mod. Phys. A 24 (2009) 1135 [arXiv:0708.3530] [SPIRES].

[65] W. Magnus, F. Oberhettinger and R.P. Soni, Formulas and theorems for the special functions of mathematical physics, Springer, U.S.A. (1966).

[66] R.S. Maier, The 192 solutions of the Heun equation, Math. Comp. 76 (2007) 811 [math/0408317].

[67] H. Suzuki, E. Takasugi and H. Umetsu, Perturbations of Kerr-de Sitter black holes and Heun's equations, Prog. Theor. Phys. 100 (1998) 491 [gr-qc/9805064] [SPIRES].

[68] H. Suzuki, E. Takasugi and H. Umetsu, Analytic solutions of Teukolsky equation in Kerr-de Sitter and Kerr-Newman-de Sitter geometries, Prog. Theor. Phys. 102 (1999) 253 [gr-qc/9905040] [SPIRES].

[69] S. Musiri and G. Siopsis, Asymptotic form of quasi-normal modes of large AdS black holes, Phys. Lett. B 576 (2003) 309 [hep-th/0308196] [SPIRES].

[70] T. Oota and Y. Yasui, Toric Sasaki-Einstein manifolds and Heun equations, Nucl. Phys. B 742 (2006) 275 [hep-th/0512124] [SPIRES]. 
[71] M. Berg and H. Samtleben, Holographic correlators in a flow to a fixed point, JHEP 12 (2002) 070 [hep-th/0209191] [SPIRES].

[72] D. Petroff, Slowly rotating homogeneous stars and the Heun equation, Class. Quant. Grav. 24 (2007) 1055 [gr-qc/0701081] [SPIRES].

[73] M. Hortacsu, Heun functions and their uses in physics, arXiv:1101.0471 [SPIRES].

[74] L. Randall and R. Sundrum, A large mass hierarchy from a small extra dimension, Phys. Rev. Lett. 83 (1999) 3370 [hep-ph/9905221] [SPIRES].

[75] S.B. Giddings, S. Kachru and J. Polchinski, Hierarchies from fluxes in string compactifications, Phys. Rev. D 66 (2002) 106006 [hep-th/0105097] [SPIRES].

[76] I.R. Klebanov and M.J. Strassler, Supergravity and a confining gauge theory: duality cascades and $\chi_{S B}$-resolution of naked singularities, JHEP 08 (2000) 052 [hep-th/0007191] [SPIRES].

[77] H. Firouzjahi and S.H.H. Tye, The shape of gravity in a warped deformed conifold, JHEP 01 (2006) 136 [hep-th/0512076] [SPIRES].

[78] T. Noguchi, M. Yamaguchi and M. Yamashita, Gravitational Kaluza-Klein modes in warped superstring compactification, Phys. Lett. B 636 (2006) 221 [hep-th/0512249] [SPIRES].

[79] G. Shiu, B. Underwood, K.M. Zurek and D.G.E. Walker, Probing the geometry of warped string compactifications at the LHC, Phys. Rev. Lett. 100 (2008) 031601 [arXiv:0705.4097] [SPIRES]. 